\title{
Forecasting the Impact of Diabetes Mellitus on Tuberculosis Disease Incidence and Mortality in India
}

Susanne F. Awad, ${ }^{1,2}$ Peijue Huangfu, ${ }^{2}$ Houssein H. Ayoub, ${ }^{1,3,4}$ Fiona Pearson, ${ }^{2}$ Soha R. Dargham, ${ }^{1}$ Julia Critchley, ${ }^{2 \#}$ and Laith J. Abu-Raddad, ${ }^{1,4,5 \# *}$

${ }^{1}$ Infectious Disease Epidemiology Group, Weill Cornell Medicine-Qatar, Cornell University, Qatar Foundation - Education City, Doha, Qatar

${ }^{2}$ Population Health Research Institute, St George’s, University of London, London, UK

${ }^{3}$ Department of Mathematics, Statistics, and Physics, Qatar University, Doha, Qatar

${ }^{4}$ Department of Healthcare Policy and Research, Weill Cornell Medicine, Cornell University, New York, New York, USA

${ }^{5}$ College of Health and Life Sciences, Hamad bin Khalifa University, Doha, Qatar

\#Both authors are joint senior authors for this study.

Word count: Abstract: 271 words, Text: 4,449 words.

Number of figures: 3

Number of tables: 2

Conflict of interest: There are no conflicts of interest

Disclose funding received for this work: Qatar National Research Fund (QNRF)

Running head: Impact of diabetes on tuberculosis

* Reprints or Correspondence: Laith J. Abu-Raddad, Ph.D., Infectious Disease Epidemiology Group, Weill Cornell Medicine-Qatar, Qatar Foundation - Education City, P.O. Box 24144, Doha, Qatar. Telephone: +(974) 4492-8321. Fax: +(974) 4492-8333. E-mail: lja2002@qatarmed.cornell.edu 


\section{ABSTRACT}

Background: In context of the rapidly expanding diabetes mellitus (DM) epidemic in India and slowly declining tuberculosis (TB) incidence, we aimed to estimate the past, current, and future impact of DM on TB epidemiology.

Methods: An age-structured TB-DM dynamical mathematical model was developed and analyzed to assess the DM-on-TB impact. The model was calibrated using a literature review and meta-analyses. The DM-on-TB impact was analyzed using population attributable fraction metrics. Sensitivity analyses were conducted by accommodating less conservative effect sizes for the TB-DM interactions, by factoring the age-dependence of the TB-DM association, and by assuming different TB disease incidence rate trajectories.

Results: In 1990, 11.4\% (95\% uncertainty interval (UI): 6.3\%-14.4\%) of new TB disease incident cases were attributed to DM. This proportion increased to 21.9\% (95\% UI: 12.1\%26.4\%) in 2017, and 33.3\% (95\% UI: 19.0\%-44.1\%) in 2050. Similarly, in 1990, 14.5\% (95\% UI: 9.5\%-18.2\%) of TB-related deaths were attributed to DM. This proportion increased to 28.9\% (95\% UI: $18.9 \%-34.1 \%$ ) in 2017, and 42.8\% (95\% UI: 28.7\%-53.1\%) in 2050. The largest impacts originated from the effects of DM on TB disease progression and infectiousness. Sensitivity analyses suggested that the impact could be even greater.

Conclusion: The burgeoning DM epidemic is predicted to become a leading driver of TB disease incidence and mortality over the coming decades. By 2050, at least one-third of TB incidence and almost half of TB mortality in India will be attributed to DM. This is likely generalizable to other Asian Pacific countries with similar TB-DM burdens. Targeting the impact 
of the increasing DM burden on TB control is critical to achieving the goal of TB elimination by 2050.

Keywords: Tuberculosis; Diabetes mellitus; Population attributable fraction; Epidemiological synergy; Epidemiological association; Mathematical modelling; India 


\section{INTRODUCTION}

Although tuberculosis (TB) remains a public health concern globally, several countries are disproportionally affected by TB [1]. India harbors the largest number of individuals with TB worldwide, with at least twice as many cases as any other country [1]. In 2016, 2.8 million incident TB disease cases (27\% of global TB incidence) and 435,000 TB deaths (26\% of global TB deaths) were estimated in India [1].

TB disease incidence is affected by key risk factors such as diabetes mellitus (DM), HIV, undernutrition, and smoking [2]. In 2017, 73 million Indians were living with DM at a prevalence of 8.8\% (95\% confidence interval [CI]: 6.7\%-10.9\%) [3]. India was projected to account for the highest number of DM cases globally by 2045 at 134 million cases [3]. With India burdened by both TB and DM, their synergetic relationship is a major public health concern. A number of TB-DM epidemiological studies have been conducted in this country [4-9], with recent data reporting high DM prevalence among TB patients [10].

DM increases the risk of TB infection [11] and disease [12-14], and has adverse impact on TB treatment outcomes (e.g., DM increases the risk of mortality during TB treatment, TB relapse, and possibly multi-drug resistant TB) [14-20]. Several biological mechanisms appear to explain the synergetic TB-DM association [21-33]. For example, the hypothesis that DM impairs the innate and adaptive immune responses, such as interferon-C (IFN-c), necessary to prevent the proliferation of TB, is supported by existing studies [13,30,34]. Studies showed that, compared to people with no DM, IFN-c levels were significantly reduced in people with DM [30], and that IFN-c levels were negatively associated with glycated hemoglobin levels [31]. 
A recent study of TB-DM interactions indicated large potential impact for DM on TB incidence including both direct (e.g., DM increasing the risk of onset of TB disease) and indirect effects (e.g., onward transmission of TB from people with and without DM) [20]. The study concluded that the impact of DM on TB epidemiology could be underestimated, if assessed using more conventional population attributable fraction (PAF) approaches such as Levin's formula [35], that capture only the direct impact of DM on TB [20].

Against this background, we aimed to estimate the past, current, and future impact of DM on TB epidemiology in India using a dynamical mathematical model. A strength of this study is that it accounts for the different pathways in which DM affects TB natural history and treatment outcomes, and incorporates a detailed quantitative assessment of the effect sizes of each of the DM-on-TB effects. The study also factors the projected rise of the DM epidemic in India over the coming decades, and assesses both the direct and indirect population impacts of DM on TB. The TB-DM model was applied to India to demonstrate the utility of our approach in a country highly burdened with both diseases, however, can be implemented in additional countries.

\section{METHODS}

We constructed an age-structured deterministic compartmental model to characterize the impact of DM on TB epidemiology in India by extending a recently developed analytical approach [20]. The model was also designed based on a recently developed conceptual framework for TB-DM interactions [20]. The model was coded and analyzed in MATLAB R2015a [36].

\section{Mathematical model}


The model is described by a system of coupled nonlinear differential equations stratifying the Indian population by age group, TB infection status, TB infection stage, TB disease form, TB treatment status, TB recovery status, and DM status. Details of the model can be found in Supplementary.

The population was stratified into 20 5-year age bands representing the age cohort 0-99 years. Upon infection, TB progression was stratified into the two stages: latent-slow TB infection (LSI) and latent-fast TB infection (LFI). TB disease was stratified into the three clinically-relevant forms: smear-positive pulmonary (SP-PTB), smear-negative pulmonary (SN-PTB), and extrapulmonary (EP-TB) [37,38]. The proportion of individuals developing each infection and disease form was age-dependent, and only the pulmonary forms were considered infectious. Treatment was assumed to last for six-months reflecting the directly-observed treatment short-course (DOTS) therapy [39].

Individuals with DM followed a distinct TB natural history from that of non-DM individualsTB natural history was modulated by specific effects of having concurrent DM (Supplementary Figure S1). Based on empirical evidence, DM was assumed to affect TB natural history and treatment outcomes through 10 different pathways [20]. The effects, their definitions, their effect sizes, and the evidence supporting them are summarized in Table 1 and discussed in Supplementary.

Briefly, compared to non-DM individuals, DM increased susceptibility to TB infection (Effect 1Susceptibility), proportion of TB infections entering LFI versus LSI states (Effect 2-Fast progression), proportion of those developing SP-PTB (versus SN-PTB) for those with pulmonary TB disease (Effect 5-Smear positivity), and TB infectiousness among those with 
pulmonary TB disease (Effect 6-Disease infectiousness). Furthermore, compared to non-DM individuals, DM increased the risk of TB-related mortality (Effect 7-TB mortality), reduced the proportion of successful treatment among those undergoing TB treatment (Effect 8-Treatment failure), delayed the resolution of TB disease (Effect 9-Recovery), and increased susceptibility to TB reinfection after recovery (Effect 10-Cured reinfection).

Amongst those with DM comparative to without, susceptibility to develop TB disease among those with LSI (Effect 3-Reactivation), and susceptibility to TB reinfection among those with LSI (Effect 4-Primary reinfection), were set as having no effect, as the impacts of these pathways were captured by Effect 2-Fast progression (Supplementary Section 3.2). Also, given heterogeneity of evidence [19], the proportion of successful treatment among those with DM undergoing TB treatment (Effect 8-Treatment failure) was set as equal to those without DM undergoing TB treatment (Supplementary Section 3.2).

\section{Data sources and model fitting}

TB natural history model parameters (in absence of DM) were based on available empirical evidence [37], or through model fitting to empirical data. Supplementary Table S1 lists the parameter values and their sources.

The key assumptions for the effect sizes of the 10 DM-on-TB effects were based mostly on pooled evidence from systematic reviews and/or meta-analyses, or derived from specific observational studies (Table 1 and Supplementary). Given heterogeneities and uncertainties around the exact effect sizes, we opted for a conservative approach whereby each effect size was modest, or set at the null value if the evidence is conflicting or not firmly established (i.e. DM 
has no effect on TB). For example, the effect size for Effect 2-Fast progression was set as derived using an effect size of only 2.00 for the TB-DM association—based on a conservative meta-analysis that pooled studies of different study designs (Supplementary Section 3.2) [12]. The effect size for Effect 7-TB mortality was based on a recent meta-analysis estimating a pooled mean crude odds ratio (OR) of 2.11 across 48 studies [19]. Despite evidence suggesting an effect for TB on DM [40], we opted not to account for this bi-directionality given that current evidence is not yet conclusive for this effect. Therefore, our estimates for the impact of the TB-DM interactions on TB epidemiology are more likely to underestimate the impact, rather than overestimate it.

The model was fitted using the following India-specific data: TB-incidence and mortality rates as reported in the World Health Organization (WHO) Global Health Observatory data repository [41], national and age-specific DM prevalence as reported by the International Diabetes Federation [3,42-46], age-specific DM prevalence distribution as reported by the nationallyrepresentative Indian Council of Medical Research-India Diabetes study [47], and demographics as reported in the database of the Population Division of the United Nations Department of Economic and Social Affairs [48]. TB contact and case-detection rates were derived by model fitting to the above data.

\section{TB-DM synergy metric}

We estimated the impact of DM on each of TB disease incidence and mortality between 1990 and 2050 by calculating the "true" PAF [20]—i.e. the proportion of each of TB incidence and mortality that is directly (etiologically) and indirectly (such as onward transmission) attributed to DM (Supplementary). In contrast with Levin's PAF [35] which only estimates the direct 
population impact of DM on TB disease, “true” PAF (below noted only as PAF) was estimated for each of TB incidence and mortality as the proportional reduction between the measures in a scenario where the synergy in the TB-DM relationship is active, compared to a scenario where the synergy is inactive. We assessed the impact of DM on TB epidemiology for each of the DM effects in combinations and individually.

\section{Uncertainty analysis}

A multivariate uncertainty analysis was conducted factoring the uncertainty in our knowledge of the DM-on-TB effect sizes (Table 1). We used Monte Carlo sampling from either the CI for the TB-DM effect sizes, or assuming (if uncertainty is not captured by CI) $\pm 25 \%$ uncertainty around the point estimates for the effect sizes. We implemented 500 uncertainty runs of the model. In each run, the values of the effect sizes were randomly selected from their specified ranges, and the model was refitted to India's country-specific data. The mean and 95\% uncertainty intervals (UI) for the PAFs were derived from the likelihood distribution generated by the uncertainty runs.

\section{Sensitivity analyses}

Given that our main estimates were generated using a conservative approach, we conducted two sensitivity analyses with less conservative effect sizes for the TB-DM interactions. In the first sensitivity analysis, we used, for Effect 2-Fast progression, the TB-DM association effect size of 3.59 based on the prospective cohort studies (Supplementary Section 3.2) [12], In the second sensitivity analysis, we used, for Effect 7-TB mortality, the effect size of 4.95 based on the pooled analysis that included studies that appropriately adjusted for confounders (Supplementary Section 3.2) [19]. 
In a third sensitivity analysis, we explored the TB-DM synergy implications by factoring the age-dependence of the TB-DM association, based on a cohort study that estimated the agespecific relative risks (RRs) of the effect of DM on TB disease [49]. In doing so, we scaled down (conservatively) the age effects reported by Kim et al [49], to reach the assumed two-fold overall RR (Supplementary Section 3.2).

In a fourth sensitivity analysis, in context of uncertainty about the future trajectory of the TB epidemic over the coming decades, we assessed the TB-DM synergy implications assuming 10 different TB disease incidence rate trajectories over the coming decades. The change in TB incidence rate at 2050, relative to the baseline model scenario, was assumed to range between $\pm 50 \%$.

In a fifth sensitivity analysis, we accounted for the age-dependency in the proportion of individuals developing each infection form (LSI versus LFI) for those aged 15 years and above. Specifically, as informed by evidence [50], we assessed the TB-DM synergy implications assuming that $25 \%$ of individuals who progress to TB infection aged 15-35 years develop LFI, while only 5\% of individuals aged 35+ years develop LFI.

In a sixth sensitivity analysis, in context of uncertainty about the level of susceptibility to TB reinfection with prior TB exposure [51,52], we assessed the TB-DM synergy implications assuming different risk levels of TB reinfection compared to first TB infection. We compared a 65\% fractional reduction (our baseline assumption [53,54]; Supplementary Table S1), no reduction, and a 35\% fractional increase in the susceptibility to TB reinfection. The different risks of reinfection were assumed for 1) individuals with LSI (that is those in latent infection), 2) individuals who successfully completed TB treatment, or 3) both individuals with LSI and those who successfully completed TB treatment. 
Additional sensitivity analyses were conducted to assess the sensitivity of model predictions to variations in the effect sizes of the DM-on-TB effects (Table 1). For each individual effect, we used the lower and upper values from either the CI for the TB-DM effect sizes, or assuming (if uncertainty is not captured by CI) $\pm 25 \%$ uncertainty around the point estimates.

\section{RESULTS}

The model fitted well the demographic (Supplementary Figure S4), TB incidence rate (Figure 1A), TB mortality rate (Figure 1C), and DM prevalence data for India (Figure 2A). From 2017 to 2050, TB disease incidence rate (defined as the ratio of total annual number of TB disease cases over total Indian population) was projected to decrease from 215 to 116 per 100,000 persons per year (Figure 1A). Meanwhile, the number of annual new (incident) cases was projected to decrease from 2.8 to 2.0 million (Figure 1B). Likewise, TB mortality rate (defined as the ratio of total annual number of TB-related deaths over total Indian population) was projected to decrease from 40.7 to 15.7 per 100,000 persons per year (Figure 1C). Meanwhile, the number of annual TB deaths was projected to decrease from 534,000 to 287,000 (Figure 1D). DM prevalence in India was projected to increase from $8.5 \%$ in 2017 to $12.1 \%$ in 2050 (Figure 2A).

While DM prevalence increased (Figure 2A) and TB incidence rate decreased (Figure 1A), the proportion of new TB incidence cases and proportion of TB-related deaths attributed to DM increased steadily (Figure 2B). In 1990, 11.4\% (95\% UI: 6.3\%-14.4\%) of new TB disease incident cases were attributed to DM (Figure 2B). This proportion increased to 21.9\% (95\% UI: $12.1 \%-26.4 \%$ ) in 2017, and was predicted to continue increasing to $33.3 \%$ (95\% UI: $19.0 \%$ 44.1\%) by 2050. Similarly, in 1990, 14.5\% (95\% UI: 9.5\%-18.2\%) of TB-related deaths were attributed to DM. This proportion increased to 28.9\% (95\% UI: 18.9\%-34.1\%) in 2017, and was predicted to continue increasing to 42.8\% (95\% UI: 28.7\%-53.1\%) by 2050 . 
Relaxing the conservative approach by using, for Effect 2-Fast progression, the TB-DM association effect size of 3.59 [12], resulted in a larger impact for the TB-DM synergy on TB disease incidence and mortality (Figure 3A). In 1990, 17.2\% of TB disease incident cases were attributed to DM, and this proportion increased to $37.0 \%$ by 2017 and $55.4 \%$ by 2050 . Meanwhile, in 1990, 19.2\% of TB-related deaths were attributed to DM, and this proportion increased to $42.1 \%$ by 2017 and $60.8 \%$ by 2050 .

Relaxing the conservative approach by using, for Effect 7-TB mortality, the effect size of 4.95 [19], resulted in a larger impact for the TB-DM synergy on TB mortality but slightly smaller impact on TB disease incidence (Figure 3B). In 1990, 7.4\% of new TB incident cases were attributed to DM, and this proportion increased to $16.2 \%$ by 2017 and $28.2 \%$ by 2050 . Meanwhile, in 1990, $14.9 \%$ of TB-related deaths were attributed to DM, and this proportion increased to $31.2 \%$ by 2017 and $47.5 \%$ by 2050 .

Exploring the TB-DM synergy implications by factoring the age-dependence of the TB-DM association, resulted in a larger impact on TB disease incidence and mortality (Figure 3A). In 1990, 13.2\% of new TB incident cases were attributed to DM, and this proportion increased to 27.9\% by 2017 and 39.2\% by 2050. Meanwhile, in 1990, 15.3\% of TB-related deaths were attributed to DM, and this proportion increased to 33.3\% by 2017 and $45.41 \%$ by 2050 .

Assessing the TB-DM synergy implications at different TB disease incidence trajectories over the coming decades resulted in minimal changes in the assessed impact of DM on TB incidence and mortality (Supplementary Figure S5). In 2050, new TB incident cases attributed to DM ranged between $26.5 \%$ and $34.5 \%$, and TB-related deaths attributed to DM ranged between $37.2 \%$ and $43.7 \%$.

Factoring the age-dependency in the proportion of individuals developing each infection form (LSI versus LFI) for those aged 15 years and above, the impact of DM on TB disease incidence 
and mortality was smaller (Figure S6). In 1990, only 6.2\% of new TB incident cases were attributed to DM, and this proportion increased to $12.6 \%$ by 2017 and $20.4 \%$ by 2050 . Meanwhile, in 1990, 8.2\% of TB-related deaths were attributed to DM, and this proportion increased to $17.7 \%$ by 2017 and $28.6 \%$ by 2050 .

Exploring the TB-DM synergy implications assuming no change in the susceptibility to TB reinfection, resulted in slightly larger impact for DM on TB disease incidence and mortality (Figure S7). By 2050, assuming no change in the susceptibility to TB reinfection among individuals who successfully completed TB treatment, with LSI, and both with LSI and those who successfully completed TB treatment, new TB incident cases attributed to DM were 33.8\%, 38.6\%, and 38.8\%, respectively, and TB-related deaths attributed to DM were $42.1 \%$, 44.9\%, and $45.7 \%$, respectively (Figure S7). Exploring the TB-DM synergy implications assuming a $35 \%$ increase in the susceptibility to TB for reinfection, resulted in a relatively larger impact for DM on TB disease incidence and mortality (Figure S7). By 2050, assuming 35\% increase in the susceptibility to TB reinfection among individuals who successfully completed TB treatment, with LSI, and both with LSI and those who successfully completed TB treatment, new TB incident cases attributed to DM were 33.5\%, 47.7\%, and 48.9\%, respectively, and TB-related deaths attributed to DM were 42.6\%, 54.3\%, and 57.1\%, respectively (Figure S7).

Table 2 shows the individual impact of each of the DM-on-TB effects at six different time points. Most effects resulted in a larger TB disease incidence and mortality, as DM prevalence increased with time. The largest impact for TB incidence was for Effect 2-Fast progression followed by Effect 6-Infectiousness (Table 2A). The proportion of TB incidence attributed to Effect 2-Fast progression increased from $8.7 \%$ in 1990 to $25.1 \%$ by 2050 . The proportion of TB incidence attributed to Effect 6-Disease infectiousness increased from 4.5\% in 1990 to $14.8 \%$ by 2050. The largest impact for TB mortality was also for Effect 2-Fast progression followed by Effect 6-Infectiousness (Table 2B). The proportion of TB-related deaths attributed to Effect 2- 
Fast progression increased from $9.9 \%$ in 1990 to $28.5 \%$ by 2050 . The proportion of TB-related deaths attributed to Effect 6-Disease infectiousness increased from 4.3\% in 1990 to $14.4 \%$ by 2050.

Effect 7-TB mortality increased TB-related deaths from 2.1\% in 1990 to $10.3 \%$ by 2050, but it reduced TB disease incidence with less TB trasnmission (due to the premature death of persons with TB disease). The impact of Effect 5-Smear positivity and Effect 10-Cured reinfection on both TB incidence and mortality changed in direction with time-a consequence of a complex interplay between TB enhanced transmission, premature death of TB disease cases, and demographic factors relating to DM age-specific prevalence distribution and TB exposure risk variation in successive birth cohorts.

\section{DISCUSSION}

We provided a comprehensive quantitative assessment of the impact of DM on TB epidemiology in India, a country heavily burdened by both diseases. Anchored on a solid foundation of current empirical evidence, the assessment accounted for both direct and indirect impacts, and factored the different effects by which DM can affect TB natural history and treatment outcomes. As DM prevalence increased and TB disease incidence declined, DM was predicted to play a major and growing role in TB epidemiology. While in 1990 only one in 10 TB disease cases was attributed to DM, currently one in five is attributed to DM, and by 2050, one in three will be attributed to DM. While in 1990 only one in seven TB-related deaths was attributed to DM, currently nearly one in three is attributed to DM, and by 2050, nearly one in two will be attributed to DM. These findings highlight how DM could be emerging as the leading driver of TB incidence and mortality in India, and likely elsewhere.

The results support growing evidence highlighting the increasing role of DM on TB epidemiology [2,55,56], but also suggest that DM impact could be underestimated. We 
investigated DM role using a conservative approach whereby the effect size for each DM-on-TB effect was set at its lowest or null value. Setting effect sizes based on best quality evidence, resulted in even larger impact of DM on TB, particularly so for TB mortality—half of TB disease cases and TB-related deaths could be attributed to DM by 2050 (Figure 3).

Although the clinical effects of DM on TB treatment outcomes have been widely discussed and researched [19], the population impact has been less investigated but shown in this study to play an influential role (such as that of Effect 7-TB mortality). However, most of the impact of DM on TB was driven by the effects of DM on TB natural history-in particular Effect 2-Fast progression and Effect 6-Disease infectiousness (Table 2). These findings suggest that intervention strategies should target DM patients before onset of TB disease. The populationlevel impacts of different intervention strategies, such as screening, case-finding, and intensified treatment, need to be investigated factoring the different DM-on-TB effects.

Our findings demonstrate that substantial reductions in TB disease incidence and mortality in India, and likely in the countries burdened by both TB and DM, are difficult to achieve without focusing on the high-level determinants and risk factors for TB including DM, as stressed in the WHO’s post-2015 TB strategy [57] and in The Collaborative Framework for Care and Control of Tuberculosis and Diabetes launched in 2011 by the WHO and International Union Against Tuberculosis and Lung Disease (The Union) [14], and as reinforced and expanded by the joint Union’s and World Diabetes Foundation’s “2014 Call for Action” [58] and the TB-DM Bali Declaration in 2015 [59]. Indeed, only a country-by-country approach, following the concept of “know your epidemic" for managing TB, may advance TB efforts towards TB elimination by 2050. While historically TB has been a general population infection and disease, its epidemiology could be transitioning into a new era driven by the dynamics of this infection in high risk populations such people living with DM. 
Our study has limitations mostly related to incomplete knowledge of the TB-DM epidemiology. We included different DM-on-TB effects based on extensive literature review and meta-analyses of existing data (Supplementary Section 3.2), but we may have overlooked effects not yet supported by evidence. For example, Effect 6-Disease infectiousness is an effect that has not been directly investigated in the literature, but seems to have a major population-level impact on TB epidemiology through its effect on the onward transmission of the infection. The parametrization of Effect 6-Disease infectiousness was based on biologically-motivated plausible assumptions that need to be investigated in details through further epidemiological/biological studies.

Evidence suggests heterogeneities and uncertainties around the exact effect sizes of several effects. For example, not all risk estimates were available by age strata, though age could be an important factor in determining the population impact of DM on TB. Moreover, even though evidence supports an increased risk of developing TB disease for those with DM [12], it does not differentiate the precise biological mechanism(s) of whether DM is acting through Effect 2-Fast progression, Effect 3-Reactivation, and/or Effect 4-Primary reinfection.

Our conclusion is predicated upon the assumption that the effect of DM on TB is causal. While strongly plausible, the scale of TB-DM biological/epidemiological synergy is not completely certain. The association could be affected by confounders, which are not controlled for given the very complex overlap and interactions between TB and DM. For example, the TB-DM interaction is paradoxical; while DM is known to be associated with obesity [60], TB is reportedly associated with low body mass index [61].

We did not include all factors that may influence the impact of DM on TB, or the factors that may affect directly each of TB or DM burdens individually [12,62-64]. For example, the impact of HIV as a co-factor [62-64] was not incorporated. However, despite the potential public health 
implications, HIV prevalence is relatively low in India at less than 1.0\% [65], hence, probably minimally affecting our results and conclusions.

We modeled TB's natural history and dynamics based on the canonical approach in the literature [37,66], but TB's complex natural history remains insufficiently-understood [50]. For instance, based on studies by Heimbeck [53,54], we assumed a proportional reduction in the susceptibility to TB reinfection with prior TB exposure (i.e., acquired protective immunity), however, this immunity may be explained by selection bias as these studies were conducted among individuals who may not have been representative of the wider population [52]. Other evidence suggests a higher risk of reinfection rather than protective immunity [51]. Moreover, though we assumed that the proportion of individuals developing LSI versus LFI was age dependent, this was assumed for only children versus adults, but the variable age dependence perhaps affects also the adult population [50].

We did not factor the effect of intermediate hyperglycemia (pre-DM) on TB, which may enhance the impact of DM on TB $[12,67]$. We only included the DM-on-TB effects, but the links between the two diseases could be bi-directional [40]. Last but not least, the impact of DM on TB depends on the trajectory of the TB epidemic over the coming decades, but this trajectory may change substantially with roll-out and scale-up of interventions in upcoming years [1].

Despite these limitations, our study has several strengths. Our model includes ten different effects in which DM affects TB natural history and treatment outcomes, incorporates a detailed quantitative assessment of the effect sizes for each effect, is age stratified to reflect the agespecific trends, and assesses both the direct and indirect population impacts of DM on TB. In addition, most of the potential limitations are likely to lead to underestimation rather than overestimation of the impact of DM on TB. 
We also conducted sensitivity analyses to explore the potential impact of several mentioned limitations, and these analyses confirmed our results, or suggested that the impact could be underestimated (Figure 3 and Supplementary Figures S5 and S7), or slightly overestimated (Supplementary Figure S6). Furthermore, our sensitivity analyses demonstrated that our results are most sensitive to Effect 2-Fast progression, Effect 6-Disease infectiousness, and Effect 1Susceptibility (Supplementary Figure S9), as expected given the impact of these effects on TBepidemiology (Table 2). Otherwise, our results were largely insensitive to variations in the rest of explored effects (Supplementary Figure S9). We further conducted a multivariate uncertainty analysis by factoring the uncertainty in model parameters, and the uncertainty intervals of the model outcomes affirmed the validity of our predictions (Supplementary Figure S8). Finally, it bears notice that the aim of the present analysis was to assess the epidemiological implications of the TB-DM interactions focusing on the core interaction effects and at the national level. Thus, we resorted to a parsimonious model structure presenting “average” impact estimates of DM on TB, rather than stratified estimates for specific population strata.

In conclusion, the burgeoning DM epidemic in India is predicted to become a leading driver of TB disease incidence and mortality over the coming decades in India and possibly elsewhere. At present, one in five TB disease cases is attributed to DM, and by 2050, one in three will be attributed to DM. Nearly one in three TB-related deaths is attributed to DM currently, and by 2050, nearly one in two will be attributed to DM. The slowly declining TB incidence, in context of rapidly expanding DM epidemic in multiple countries, could be driving a major turn in TB epidemiology. Targeting the impact of the increasing DM burden on TB control is critical to achieving the goal of TB elimination by 2050. 


\section{ACKNOWLEDGEMENTS}

This publication was made possible by NPRP grant number 7-627-3-167 from the Qatar National Research Fund (a member of Qatar Foundation). The findings achieved herein are solely the responsibility of the authors. The authors are also grateful for infrastructure support provided by the Biostatistics, Epidemiology, and Biomathematics Research Core at Weill Cornell MedicineQatar.

\section{AUTHORS AND CONTRIBUTORS}

SFA co-conceived and co-designed the study, programmed the model, conducted the modeling analyses, and wrote the first draft of the article. LJA and JC obtained the funding, led the study, co-conceived and co-designed the study, and contributed to conduct of the analyses. $\mathrm{PH}$, FP, and SRD contributed to some statistical analyses related to model parametrization. HHA contributed to model development. All authors contributed to study development, analysis and interpretation of results, and writing of the article.

\section{CONFLICT OF INTEREST}

We declare that we have no conflict of interest to disclose. 


\section{REFERENCES}

1 World Health Organization. Global tuberculosis report 2016 (Available from: http://apps.who.int/iris/bitstream/10665/250441/1/9789241565394-eng.pdf?ua=1; accessed on May 2017). 2016

2 Lonnroth K, Castro KG, Chakaya JM, Chauhan LS, Floyd K, Glaziou P, et al. Tuberculosis control and elimination 2010-50: cure, care, and social development. Lancet. 2010;375:1814-29.

3 International Diabetes Federation. IDF Diabetes Atlas. Eighth edition. Brussels, Belgium (Available at: http://www.diabetesatlas.org, accessed Dec. 2017). 2017.

$4 \quad$ K VN, Duraisamy K, Balakrishnan S, M S, S JS, Sagili KD, et al. Outcome of tuberculosis treatment in patients with diabetes mellitus treated in the revised national tuberculosis control programme in Malappuram District, Kerala, India. PLoS One. 2013;8:e76275.

5 Viswanathan V, Kumpatla S, Aravindalochanan V, Rajan R, Chinnasamy C, Srinivasan R, et al. Prevalence of diabetes and pre-diabetes and associated risk factors among tuberculosis patients in India. PLoS One. 2012;7:e41367.

6 Viswanathan V, Vigneswari A, Selvan K, Satyavani K, Rajeswari R, Kapur A. Effect of diabetes on treatment outcome of smear-positive pulmonary tuberculosis--a report from South India. Journal of diabetes and its complications. 2014;28:162-5.

7 Balakrishnan S, Vijayan S, Nair S, Subramoniapillai J, Mrithyunjayan S, Wilson N, et al. High diabetes prevalence among tuberculosis cases in Kerala, India. PLoS One. 2012;7:e46502.

8 Burki T. Why India should worry about a coepidemic of diabetes and tuberculosis. BMJ. 2015;350:h111.

9 Singh SP, Singh SP, Kishan J, Kaur S, Ramana S. Association of tuberculosis and diabetes Mellitus: an analysis of 1000 consecutively admitted cases in a tertiary care hospital of North India. Pan Afr Med J. 2016;24:4.

10 Ministry of Health \& Family Welfare (Supported by WHO Country Office for India). National framework for joint TB-Diabetes collaborative activities, Revised National Tuberculosis Control Programme (RNTCP) (Available at: https://tbcindia.gov.in/WriteReadData/National\%20framework\%20for\%20joint\%20TB\%20diabe tes\%2023\%20Aug\%202017.pdf; Accessed Dec. 2018). 2017.

11 Martinez L, Zhu L, Castellanos ME, Liu Q, Chen C, Hallowell BD, et al. Glycemic Control and the Prevalence of Tuberculosis Infection: A Population-based Observational Study. Clinical infectious diseases : an official publication of the Infectious Diseases Society of America. 2017.

12 Al-Rifai RH, Pearson F, Critchley JA, Abu-Raddad LJ. Association between diabetes mellitus and active tuberculosis: A systematic review and meta-analysis. PLoS One. 2017;12:e0187967.

13 Jeon $\mathrm{CY}$, Murray MB. Diabetes mellitus increases the risk of active tuberculosis: a systematic review of 13 observational studies. PLoS medicine. 2008;5:e152.

14 World Health Organization, International Union Against Tuberculosis and Lung Disease. Collaborative framework for care and control of tuberculosis and diabetes. Switzerland: World Health Organization, 2011 16/11/2013. Report No.

15 Baker MA, Harries AD, Jeon CY, Hart JE, Kapur A, Lönnroth K, et al. The impact of diabetes on tuberculosis treatment outcomes: A systematic review. BMC Medicine. 2011;9:81-. 
Faurholt-Jepsen D, Range N, PrayGod G, Jeremiah K, Faurholt-Jepsen M, Aabye MG, et al. Diabetes is a strong predictor of mortality during tuberculosis treatment: a prospective cohort study among tuberculosis patients from Mwanza, Tanzania. Tropical medicine \& international health : TM \& IH. 2013;18:822-9.

17 Stevenson CR, Critchley JA, Forouhi NG, Roglic G, Williams BG, Dye C, et al. Diabetes and the risk of tuberculosis: a neglected threat to public health? Chronic illness. 2007;3:228-45.

18 Faurholt-Jepsen D, Range N, Praygod G, Kidola J, Faurholt-Jepsen M, Aabye MG, et al. The role of diabetes co-morbidity for tuberculosis treatment outcomes: a prospective cohort study from Mwanza, Tanzania. BMC infectious diseases. 2012;12:165.

19 Huangfu P, Ugarte-Gil C, Golub J, Pearson F, Critchley J. The effects of diabetes on tuberculosis treatment outcomes: an updated systematic review and meta-analysis Under Review. 2017.

20 Awad SF, Dargham SR, Omori R, Pearson F, Critchley JA, Abu-Raddad LJ. Analytical Insights into the Impact of Diabetes Mellitus on Tuberculosis Epidemiology. Under review. 2019.

21 Casqueiro J, Casqueiro J, Alves C. Infections in patients with diabetes mellitus: A review of pathogenesis. Indian J Endocrinol Metab. 2012;16 Suppl 1:S27-36.

22 Geerlings SE, Hoepelman AIM. Immune dysfunction in patients with diabetes mellitus (DM). Fems Immunology and Medical Microbiology. 1999;26:259-65.

23 Peleg AY, Weerarathna T, McCarthy JS, Davis TME. Common infections in diabetes: pathogenesis, management and relationship to glycaemic control. Diabetes-Metabolism Research and Reviews. 2007;23:3-13.

24 Joshi N, Caputo GM, Weitekamp MR, Karchmer AW. Infections in patients with diabetes mellitus. N Engl J Med. 1999;341:1906-12.

25 Vardakas KZ, Siempos II, Falagas ME. Diabetes mellitus as a risk factor for nosocomial pneumonia and associated mortality. Diabetic Medicine. 2007;24:1168-71.

26 Restrepo BI, Camerlin AJ, Rahbar MH, Wang WW, Restrepo MA, Zarate I, et al. Cross-sectional assessment reveals high diabetes prevalence among newly-diagnosed tuberculosis cases. Bulletin of the World Health Organization. 2011;89:352-9.

27 Harries AD, Lin Y, Satyanarayana S, Lonnroth K, Li L, Wilson N, et al. The looming epidemic of diabetes-associated tuberculosis: learning lessons from HIV-associated tuberculosis. International Journal of Tuberculosis and Lung Disease. 2011;15:1436-44.

28 Dooley KE, Chaisson RE. Tuberculosis and diabetes mellitus: convergence of two epidemics. Lancet Infectious Diseases. 2009;9:737-46.

29 Viardot A, Grey ST, Mackay F, Chisholm D. Potential antiinflammatory role of insulin via the preferential polarization of effector T cells toward a T helper 2 phenotype. Endocrinology. 2007;148:346-53.

30 Stalenhoef JE, Alisjahbana B, Nelwan EJ, van der Ven-Jongekrijg J, Ottenhoff TH, van der Meer $\mathrm{JW}$, et al. The role of interferon-gamma in the increased tuberculosis risk in type 2 diabetes mellitus. European journal of clinical microbiology \& infectious diseases : official publication of the European Society of Clinical Microbiology. 2008;27:97-103.

31 Tsukaguchi K, Okamura H, Ikuno M, Kobayashi A, Fukuoka A, Takenaka $H$, et al. [The relation between diabetes mellitus and IFN-gamma, IL-12 and IL-10 productions by CD4+ alpha beta T cells and monocytes in patients with pulmonary tuberculosis]. Kekkaku. 1997;72:617-22.

32 Delamaire M, Maugendre D, Moreno M, Le Goff MC, Allannic H, Genetet B. Impaired leucocyte functions in diabetic patients. Diabet Med. 1997;14:29-34.

33 Rayfield EJ, Ault MJ, Keusch GT, Brothers MJ, Nechemias C, Smith H. Infection and diabetes: the case for glucose control. Am J Med. 1982;72:439-50.

34 Dooley KE, Chaisson RE. Tuberculosis and diabetes mellitus: convergence of two epidemics. Lancet Infect Dis. 2009;9:737-46. 
Levin ML. The occurrence of lung cancer in man. Acta Unio Int Contra Cancrum. 1953;9:531-41. The MathWorks, Inc. MATLAB. The language of technical computing. 8.5.0.197613 (R2015a). Natick, MA, USA: ed: The MathWorks, Inc.; 2015.

37 Abu-Raddad LJ, Sabatelli L, Achterberg JT, Sugimoto JD, Longini IM, Jr., Dye C, et al. Epidemiological benefits of more-effective tuberculosis vaccines, drugs, and diagnostics. Proceedings of the National Academy of Sciences of the United States of America. 2009;106:13980-5.

38 Dye C, Garnett GP, Sleeman K, Williams BG. Prospects for worldwide tuberculosis control under the WHO DOTS strategy. Directly observed short-course therapy. Lancet. 1998;352:1886-91.

39 World Health Organization. What is DOTS? A Guide to Understanding the WHO-recommended TB Control Strategy Known as DOTS (available at: http://apps.who.int/iris/bitstream/10665/65979/1/WHO CDS CPC TB 99.270.pdf. Accessed February 2018). Geneva: World Health Organization, 1999.

40 Young F, Wotton CJ, Critchley JA, Unwin NC, Goldacre MJ. Increased risk of tuberculosis disease in people with diabetes mellitus: record-linkage study in a UK population. Journal of epidemiology and community health. 2012;66:519-23.

41 World Health Organization. WHO Global Health Observatory Data Repository, (available at: http://apps.who.int/gho/data/node.main). 2017. Accessed.

42 International Diabetes Federation. IDF Diabetes Atlas. 3th edition. Brussels, Belgium (available at: https://www.idf.org/sites/default/files/Diabetes-Atlas-3rd-edition.pdf; accessed on December 2015). 2006.

43 International Diabetes Federation. IDF diabetes atlas, sixth edition (available at: www.idf.org/diabetesatlas). International Diabetes Federation, 2013.

44 International Diabetes Federation. IDF Diabetes Atlas. 7th edition. Brussels, Belgium (Available at:http://www.diabetesatlas.org; accessed on September 2016). 2016.

45 Guariguata L, Whiting DR, Hambleton I, Beagley J, Linnenkamp U, Shaw JE. Global estimates of diabetes prevalence for 2013 and projections for 2035. Diabetes research and clinical practice. 2014;103:137-49.

46 Whiting DR, Guariguata L, Weil C, Shaw J. IDF diabetes atlas: global estimates of the prevalence of diabetes for 2011 and 2030. Diabetes research and clinical practice. 2011;94:311-21.

47 Anjana RM, Deepa M, Pradeepa R, Mahanta J, Narain K, Das HK, et al. Prevalence of diabetes and prediabetes in 15 states of India: results from the ICMR-INDIAB population-based crosssectional study. The lancet Diabetes \& endocrinology. 2017;5:585-96.

48 United Nations Department of Economic and Social Affairs, Population Division, Population Estimates and Projections Section. World population prospects, the 2012 revision. 2012. available: http://esa.un.org/wpp/Excel-Data/population.htm

49 Kim SJ, Hong YP, Lew WJ, Yang SC, Lee EG. Incidence of pulmonary tuberculosis among diabetics. Tubercle and Lung Disease. 1995;76:529-33.

50 Nico J.D. Nagelkerke. Courtesans and consumption. How sexually transmitted infections drive tuberculosis epidemics. The Netherlands (www.eburon.nl). Eburon, Delft. ISBN: 978-90-5972603-1 (paperback), ISBN: 978-90-5972-604-8 (ebook); 2012.

51 Verver S, Warren RM, Beyers N, Richardson M, van der Spuy GD, Borgdorff MW, et al. Rate of reinfection tuberculosis after successful treatment is higher than rate of new tuberculosis. American journal of respiratory and critical care medicine. 2005;171:1430-5.

52 Nagelkerke NJ, de Vlas SJ, Mahendradhata Y, Ottenhoff TH, Borgdorff M. The search for a tuberculosis vaccine: an elusive quest? Tuberculosis (Edinburgh, Scotland). 2006;86:41-6.

53 Heimbeck J. Incidence of tuberculosis in young adult women, with special reference to employment. British Journal of Tuberculosis. 1938;32:154-66. 
Heimbeck J. Immunity to tuberculosis. Archives of Internal Medicine. 1928;41:336-42. tuberculosis: the impact of the diabetes epidemic on tuberculosis incidence. BMC public health. 2007;7:234.

56 Pan SC, Ku CC, Kao D, Ezzati M, Fang CT, Lin HH. Effect of diabetes on tuberculosis control in 13 countries with high tuberculosis: a modelling study. The lancet Diabetes \& endocrinology. 2015;3:323-30.

57 World Health Organization. Draft global strategy and targets for tuberculosis prevention, care and control after 2015 (Available at: http://apps.who.int/gb/ebwha/pdf files/WHA67/A67 11en.pdf, accessed April 2018). 2014. International Union Against Tuberculosis and Lung Disease, World Diabetes Foundation. The Looming Co-epidemic of TB-Diabetes: A Call to Action (Available at: https://www.theunion.org/what-we-do/publications/technical/lowresolution/25383 TCB Report LR.pdf; accessed April 2018). 2014.

59 Kapur A, Harries AD, Lonnroth K, Wilson P, Sulistyowati LS. Diabetes and tuberculosis coepidemic: the Bali Declaration. The lancet Diabetes \& endocrinology. 2016;4:8-10.

60 Guh DP, Zhang W, Bansback N, Amarsi Z, Birmingham CL, Anis AH. The incidence of comorbidities related to obesity and overweight: a systematic review and meta-analysis. BMC public health. 2009;9:88.

61 Lonnroth K, Williams BG, Cegielski P, Dye C. A consistent log-linear relationship between tuberculosis incidence and body mass index. Int J Epidemiol. 2010;39:149-55.

62 Young F, Critchley JA, Johnstone LK, Unwin NC. A review of co-morbidity between infectious and chronic disease in Sub Saharan Africa: TB and diabetes mellitus, HIV and metabolic syndrome, and the impact of globalization. Globalization and health. 2009;5:9.

63 Levitt NS, Bradshaw D. The impact of HIV/AIDS on Type 2 diabetes prevalence and diabetes healthcare needs in South Africa: projections for 2010. Diabet Med. 2006;23:103-4.

64 Faurholt-Jepsen D, Range N, PrayGod G, Jeremiah K, Faurholt-Jepsen M, Aabye MG, et al. Diabetes is a strong predictor of mortality during tuberculosis treatment: a prospective cohort study among tuberculosis patients from Mwanza, Tanzania. Tropical Medicine \& International Health. 2013;18:822-9.

65 Paranjape RS, Challacombe SJ. HIV/AIDS in India: an overview of the Indian epidemic. Oral diseases. 2016;22 Suppl 1:10-4.

66 Vynnycky E, Fine PE. The natural history of tuberculosis: the implications of age-dependent risks of disease and the role of reinfection. Epidemiology and infection. 1997;119:183-201.

67 Owiti P, Keter A, Harries AD, Pastakia S, Wambugu C, Kirui N, et al. Diabetes and pre-diabetes in tuberculosis patients in western Kenya using point-of-care glycated haemoglobin. Public Health Action. 2017;7:147-54. Jan J Barendregt. Ersatz Function Overview Version 1.35 (Available at: http://www.epigear.com/index files/Ersatz\%20Function\%200verview.pdf). 2015.

69 Chang JT, Dou HY, Yen CL, Wu YH, Huang RM, Lin HJ, et al. Effect of type 2 diabetes mellitus on the clinical severity and treatment outcome in patients with pulmonary tuberculosis: a potential role in the emergence of multidrug-resistance. Journal of the Formosan Medical Association = Taiwan yi zhi. 2011;110:372-81.

70 John NN, George JM, Narmadha M. Study on the prevalence and incidence rates of diabetes mellitus in tuberculosis. International Journal of Pharmaceutical, Chemical \& Biological Sciences. 2017;7:401-6.

71 Singla R, Khan N, Al-Sharif N, Ai-Sayegh MO, Shaikh MA, Osman MM. Influence of diabetes on manifestations and treatment outcome of pulmonary TB patients. The international journal of 
tuberculosis and lung disease : the official journal of the International Union against Tuberculosis and Lung Disease. 2006;10:74-9.

72 Chiang CY, Bai KJ, Lin HH, Chien ST, Lee JJ, Enarson DA, et al. The Influence of Diabetes, Glycemic Control, and Diabetes-Related Comorbidities on Pulmonary Tuberculosis. PLoS ONE. 2015;10:e0121698.

73 Dooley KE, Tang T, Golub JE, Dorman SE, Cronin W. Impact of Diabetes Mellitus on Treatment Outcomes of Patients with Active Tuberculosis. The American Journal of Tropical Medicine and Hygiene. 2009;80:634-9.

74 Restrepo BI, Fisher-Hoch SP, Crespo JG, Whitney E, Perez A, Smith B, et al. Type 2 diabetes and tuberculosis in a dynamic bi-national border population. Epidemiology and infection. 2007;135:483-91.

75 Duangrithi D, Thanachartwet V, Desakorn V, Jitruckthai P, Phojanamongkolkij K, Rienthong S, et al. Impact of diabetes mellitus on clinical parameters and treatment outcomes of newly diagnosed pulmonary tuberculosis patients in Thailand. International journal of clinical practice. 2013;67:1199-209.

76 Suwanpimolkul G, Grinsdale JA, Jarlsberg LG, Higashi J, Osmond DH, Hopewell PC, et al. Association between diabetes mellitus and tuberculosis in United States-born and foreign-born populations in San Francisco. PLoS One. 2014;9:e114442. 
Table 1. Key assumptions for the effects of diabetes mellitus (DM) on tuberculosis (TB) natural

history and treatment outcomes.

\begin{tabular}{|c|c|c|c|c|c|}
\hline Effect & Description & Effects size & $\begin{array}{l}\text { Range for } \\
\text { uncertainty analysis }\end{array}$ & $\begin{array}{l}\text { Distribution used for } \\
\text { uncertainty analysis }\end{array}$ & Sources \\
\hline \multicolumn{6}{|c|}{ Effects of DM on TB natural history (TB infection and TB disease) } \\
\hline $\begin{array}{l}\text { Effect 1- } \\
\text { Susceptibility }\end{array}$ & $\begin{array}{l}\text { DM increases susceptibility to TB } \\
\text { infection }\end{array}$ & 1.50 & $1.0-2.2$ & Lognormal [68] & {$[11]$} \\
\hline $\begin{array}{l}\text { Effect 2-Fast } \\
\text { progression }\end{array}$ & $\begin{array}{l}\text { DM increases the proportion of TB } \\
\text { infections entering latent-fast state as } \\
\text { opposed to latent-slow state }\end{array}$ & Fitting parameter & - & Lognormal [68] & $\begin{array}{l}\text { To fit the measured meta- } \\
\text { analytically pooled TB- } \\
\text { DM association of } 2.00 \\
(95 \% \text { CI: } 1.78-2.24)[12]\end{array}$ \\
\hline $\begin{array}{l}\text { Effect 3- } \\
\text { Reactivation }\end{array}$ & $\begin{array}{l}\text { DM increases the rate of developing TB } \\
\text { disease among those with latent TB } \\
\text { infection }\end{array}$ & 1.00 (no effect) & - & - & \\
\hline $\begin{array}{l}\text { Effect } 4 \text {-Latent } \\
\text { reinfection }\end{array}$ & $\begin{array}{l}\text { DM increases the susceptibility to TB } \\
\text { reinfection among those with latent- } \\
\text { slow TB infection }\end{array}$ & 1.00 (no effect) & - & - & \\
\hline $\begin{array}{l}\text { Effect 5-Smear } \\
\text { positivity }\end{array}$ & $\begin{array}{l}\text { DM increases the proportion of new } \\
\text { PTB }^{\#} \text { disease cases progressing to SP- } \\
\text { PTB }^{*} \text { as opposed to SN-PTB }\end{array}$ & $\begin{array}{l}\kappa=1.25 \\
\kappa^{\prime}=0.67\end{array}$ & $\begin{array}{l}\kappa=[1.20-1.32] \\
\kappa^{\prime}=[0.65-0.68]\end{array}$ & Normal & $\begin{array}{l}\text { Estimated based on meta- } \\
\text { analysis of existing data } \\
\text { and Supplementary } \\
\text { Equation S1-S3 } \\
\text { (Supplementary Section } \\
\text { 3.2) }\end{array}$ \\
\hline $\begin{array}{l}\text { Effect 6-Disease } \\
\text { infectiousness }\end{array}$ & $\begin{array}{l}\text { DM increases the infectiousness of PTB } \\
\text { (SP-PTB and SN-PTB) for untreated } \\
\text { and treated TB disease cases }\end{array}$ & 1.46 & $\pm 25 \%$ & Uniform & $\begin{array}{l}\text { Estimated based on } \\
\text { weighted average of } \\
\text { existing data [69-75] }\end{array}$ \\
\hline $\begin{array}{l}\text { Effect } 7-T B \\
\text { mortality }\end{array}$ & $\begin{array}{l}\text { DM increases the hazard of TB-related } \\
\text { mortality for untreated and treated TB } \\
\text { disease cases }\end{array}$ & 2.11 & $1.76-2.51$ & Lognormal [68] & $\begin{array}{l}\text { Estimated based on meta- } \\
\text { analysis of existing data } \\
\text { [19] }\end{array}$ \\
\hline \multicolumn{6}{|c|}{ Effects of DM on TB treatment outcomes } \\
\hline $\begin{array}{l}\text { Effect } 8 \text { - } \\
\text { Treatment } \\
\text { failure }\end{array}$ & $\begin{array}{l}\text { DM reduces the proportion of } \\
\text { successful treatment (through increased } \\
\text { risk of treatment failure and MDR-TB }{ }^{\sharp} \text { ) }\end{array}$ & 1.00 (no effect) & - & - & $\begin{array}{l}\text { Estimated based on meta- } \\
\text { analysis of existing data } \\
\text { (Supplementary Section } \\
\text { 3.2) }\end{array}$ \\
\hline $\begin{array}{l}\text { Effect 9- } \\
\text { Recovery }\end{array}$ & $\begin{array}{l}\text { DM reduces the rate of TB recovery } \\
\text { (i.e. prolongs the recovery time) for } \\
\text { those who recover naturally or due to } \\
\text { treatment }\end{array}$ & 0.82 & $\pm 25 \%$ & Uniform & $\begin{array}{l}\text { Estimated based on } \\
\text { weighted average of } \\
\text { existing data }[6,69,76]\end{array}$ \\
\hline $\begin{array}{l}\text { Effect 10-Cured } \\
\text { reinfection }\end{array}$ & $\begin{array}{l}\text { DM increases susceptibility to TB } \\
\text { reinfection among those treated or } \\
\text { recovered from TB disease }\end{array}$ & 1.80 & $1.40-2.30$ & Lognormal [68] & $\begin{array}{l}\text { Estimated based on meta- } \\
\text { analysis of existing data } \\
\text { [19] }\end{array}$ \\
\hline
\end{tabular}

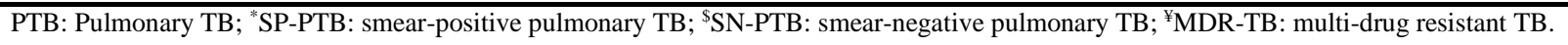


Table 2. The epidemiologic impact of each of the individual diabetes mellitus (DM) effects on tuberculosis (TB) natural history and treatment outcomes as measured by the population attributable fraction.

\begin{tabular}{|c|c|c|c|c|c|c|c|c|c|c|}
\hline \multirow{2}{*}{$\begin{array}{l}\text { Time } \\
\text { (year) }\end{array}$} & \multirow{2}{*}{$\begin{array}{c}\text { TB disease } \\
\text { incident cases }\end{array}$} & \multirow{2}{*}{$\begin{array}{c}\text { DM prevalence } \\
\text { (\%) }\end{array}$} & \multicolumn{8}{|c|}{ Population attributable fraction (\%) } \\
\hline & & & $\begin{array}{c}\text { Effect 1- } \\
\text { Susceptibility }\end{array}$ & $\begin{array}{l}\text { Effect 2-Fast } \\
\text { progression }\end{array}$ & $\begin{array}{c}\text { Effect 5-Smear } \\
\text { positivity }^{\#}\end{array}$ & $\begin{array}{c}\text { Effect 6-Disease } \\
\text { infectiousness }\end{array}$ & $\begin{array}{l}\text { Effect 7-TB } \\
\text { mortality* }\end{array}$ & $\begin{array}{l}\text { Effect 9- } \\
\text { Recovery }\end{array}$ & $\begin{array}{c}\text { Effect 10-Cured } \\
\text { reinfection }\end{array}$ & All effects \\
\hline 1990 & $3,077,706$ & 3.7 & 1.5 & 8.7 & 0.9 & 4.5 & $(2.0)$ & 0.2 & 0.9 & 11.4 \\
\hline 2010 & $2,974,690$ & 7.3 & 3.1 & 15.5 & 1.2 & 8.2 & $(3.2)$ & 0.4 & 1.7 & 20.2 \\
\hline 2020 & $2,775,774$ & 8.9 & 4.1 & 17.0 & 1.0 & 9.2 & (3.1) & 0.5 & 1.7 & 22.4 \\
\hline 2030 & $2,528,050$ & 10.2 & 5.6 & 18.3 & 0.5 & 10.2 & $(3.0)$ & 0.5 & 1. 6 & 24.3 \\
\hline 2040 & $2,274,153$ & 11.2 & 7.7 & 20.8 & $(0.2)$ & 11.9 & (3.4) & 0.6 & 1.4 & 27.8 \\
\hline 2050 & $2,038,877$ & 12.1 & 10.8 & 25.1 & (1.1) & 14.8 & $(4.2)$ & 0.8 & 1.5 & 33.3 \\
\hline
\end{tabular}

\begin{tabular}{|c|c|c|c|c|c|c|c|c|c|c|}
\hline \multirow{2}{*}{$\begin{array}{l}\text { Time } \\
\text { (year) }\end{array}$} & \multirow{2}{*}{$\begin{array}{c}\text { TB-related } \\
\text { deaths }\end{array}$} & \multirow{2}{*}{$\begin{array}{c}\text { DM prevalence } \\
(\%)\end{array}$} & \multicolumn{8}{|c|}{ Population attributable fraction (\%) } \\
\hline & & & $\begin{array}{c}\text { Effect 1- } \\
\text { Susceptibility }\end{array}$ & $\begin{array}{l}\text { Effect 2-Fast } \\
\text { progression }\end{array}$ & $\begin{array}{c}\text { Effect 5-Smear } \\
\text { positivity }^{\#}\end{array}$ & $\begin{array}{c}\text { Effect 6-Disease } \\
\text { infectiousness }\end{array}$ & $\begin{array}{c}\text { Effect 7-TB } \\
\text { mortality }\end{array}$ & $\begin{array}{l}\text { Effect 9- } \\
\text { Recovery }\end{array}$ & $\begin{array}{c}\text { Effect 10-Cured } \\
\text { reinfection }\end{array}$ & All effects \\
\hline 1990 & 802,790 & 3.7 & 1.7 & 9.9 & 0.8 & 4.3 & 2.1 & 0.8 & 1.0 & 14.5 \\
\hline 2010 & 586,316 & 7.3 & 3.6 & 18.0 & 1.0 & 8.0 & 4.7 & 1.7 & 1.9 & 26.3 \\
\hline 2020 & 491,094 & 8.9 & 4.8 & 20.0 & 0.5 & 9.0 & 6.4 & 2.2 & 2.0 & 29.9 \\
\hline 2030 & 412,743 & 10.2 & 6.6 & 21.5 & $(0.4)$ & 9.9 & 8.1 & 2.6 & 1.8 & 32.9 \\
\hline 2040 & 350,711 & 11.2 & 9.0 & 24.1 & $(1.7)$ & 11.6 & 9.4 & 3.1 & 1.7 & 37.0 \\
\hline 2050 & 302,349 & 12.1 & 12.4 & 28.5 & (3.3) & 14.4 & 10.3 & 3.7 & 1.7 & 42.7 \\
\hline
\end{tabular}

*The impact of Effect 7-TB mortality on TB incidence is negative due to the fact that Effect 7-TB mortality reduced TB disease incidence due to the premature death of persons with TB disease.


The impact of Effect 5-Smear positivity and Effect 10-Cured reinfection on both TB incidence and mortality changed in direction with time as a consequence of a complex interplay between TB enhanced transmission, premature death of persons with TB disease, and demographic factors relating to DM age-specific prevalence distribution and TB exposure risk variation in successive birth 
Figure 1. Model projections for (A) tuberculosis (TB) disease incidence rate, (B) number of annual new (incident) TB disease cases,

(C) TB mortality rate, and (D) number of annual TB deaths, in India between 1990 and 2050. The red astericks in panels A and C are the data provided by the World Health Organization's Global Health Observatory data repository [41].
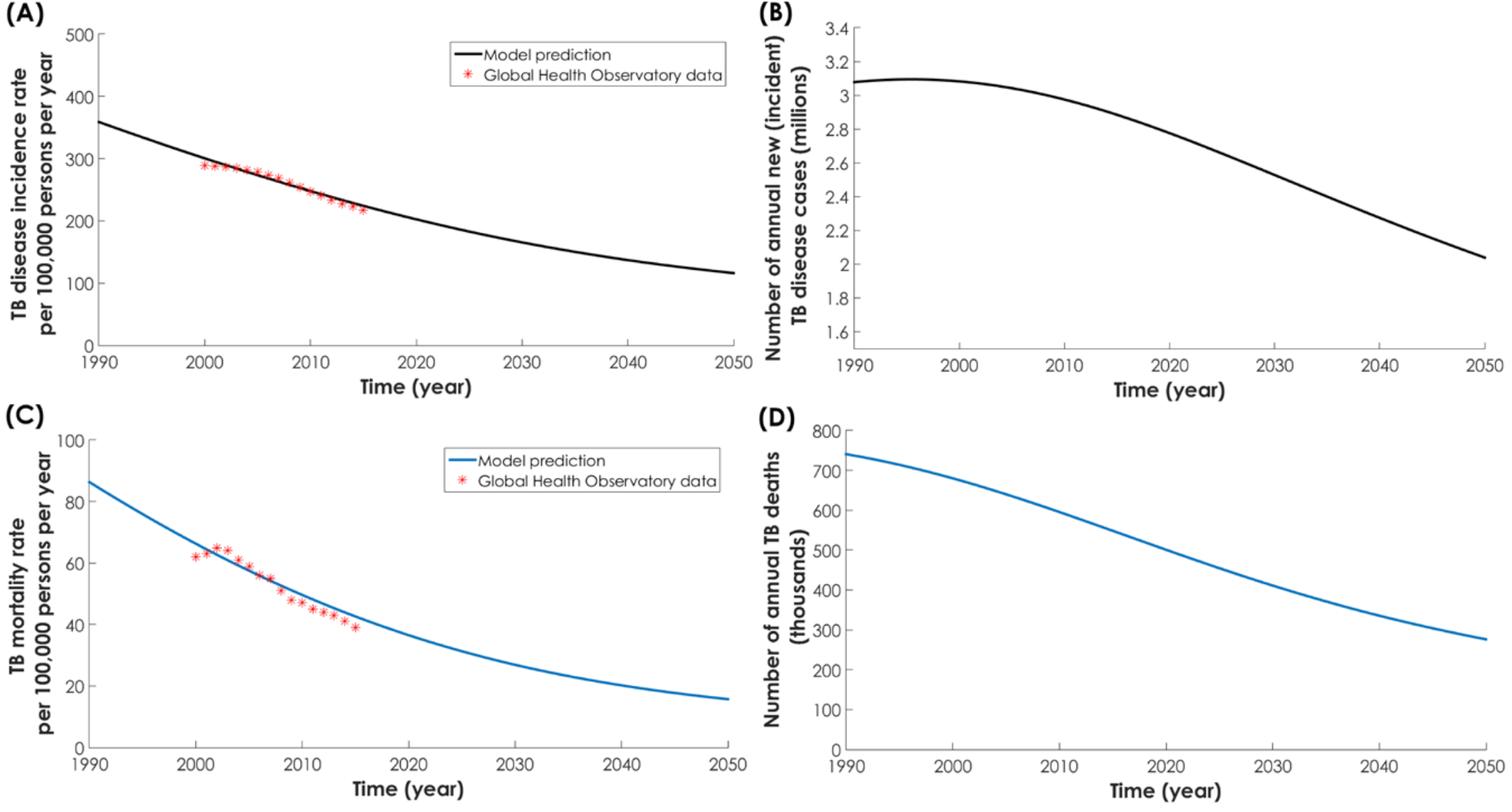
Figure 2. (A) Model projections for diabetes mellitus (DM) prevalence in India between 1990 and 2050. (B) Model predictions for the proportion of tuberculosis (TB) disease incident (solid black line) and mortality (dashes blue line) cases attributed to DM in India between 1990 and 2050. The blue and red astericks in panel A are DM prevalence data provided by the International Diabetes Federation (IDF) [44].

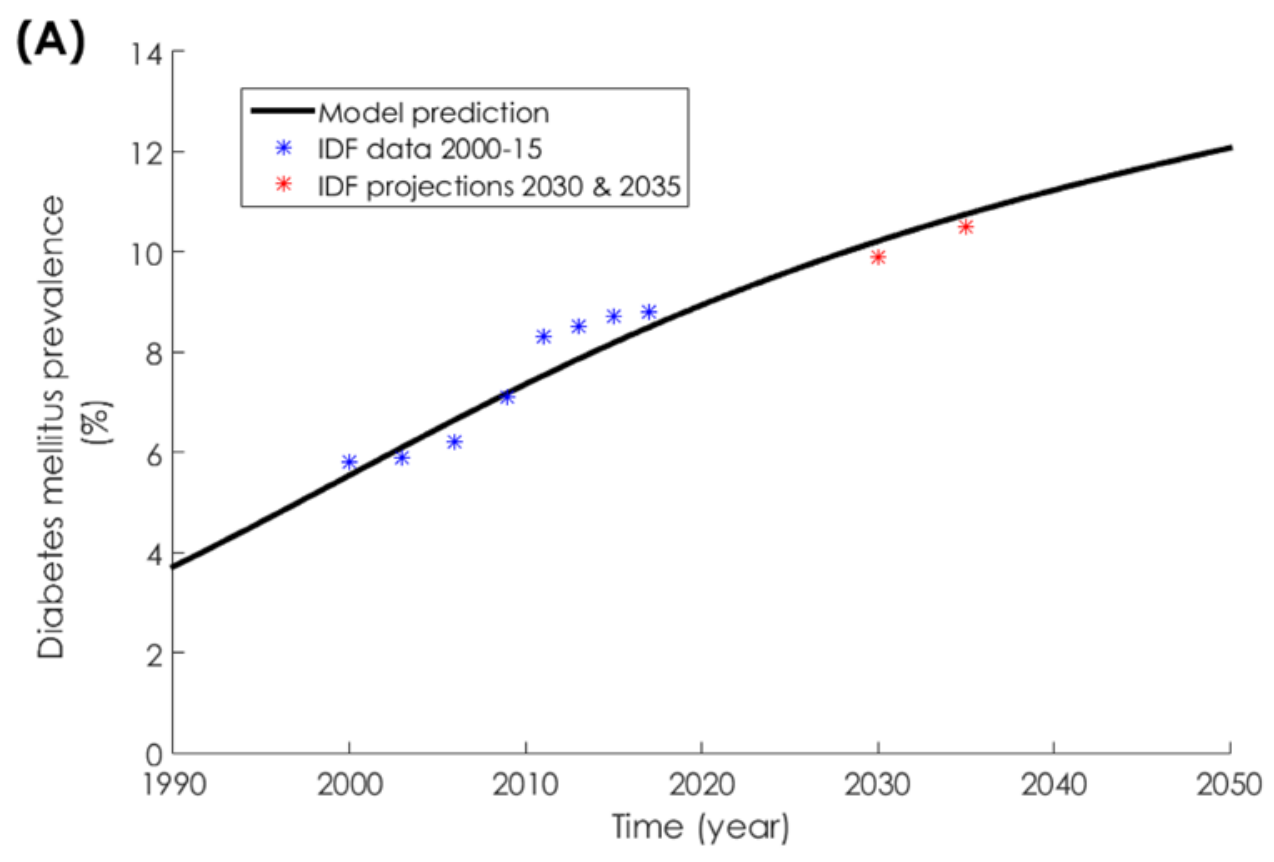

(B)

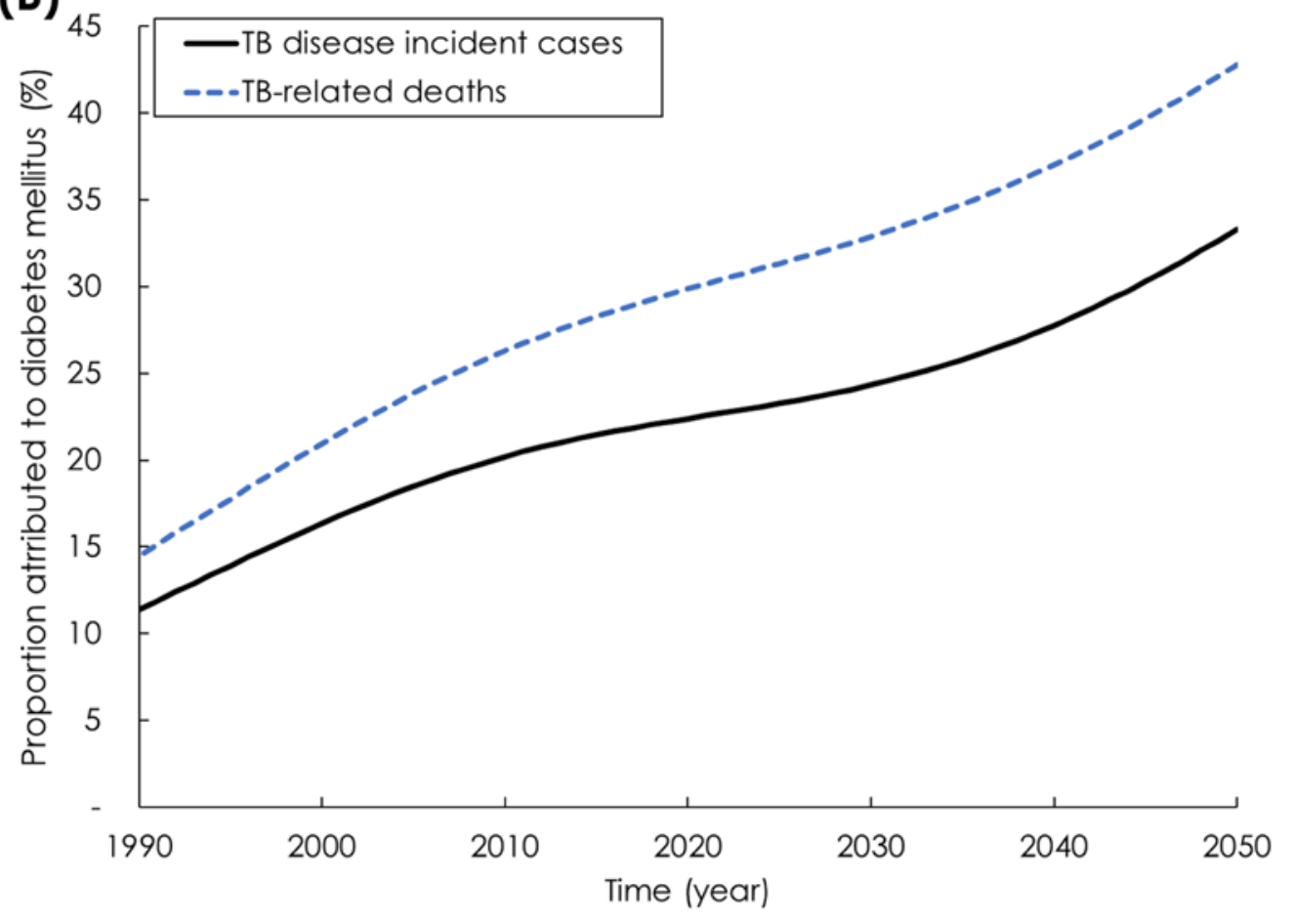


Figure 3. Sensitivity analyses. Model predictions for the proportion of tuberculosis (TB) disease incident (solid black line) and mortality (dashes blue line) cases attributed to DM in India between 1990 and 2050 assuming (A) TB-DM association effect size of 3.59 based on pooling the data only from the prospective cohort studies (Effect 2-Fast progression, Supplementary Section 3.2) [12], (B) Effect 7-TB mortality effect size of 4.95 based on the pooled analysis that included only studies that appropriately adjusted for confounders (Supplementary Section 3.2) [15], and (C) age-dependence in the TB-DM association based on a cohort study that estimated the age-specific relative risks of the effect of DM on TB disease (Effect 2-Fast progression, Supplementary Section 3.2) [49]. 

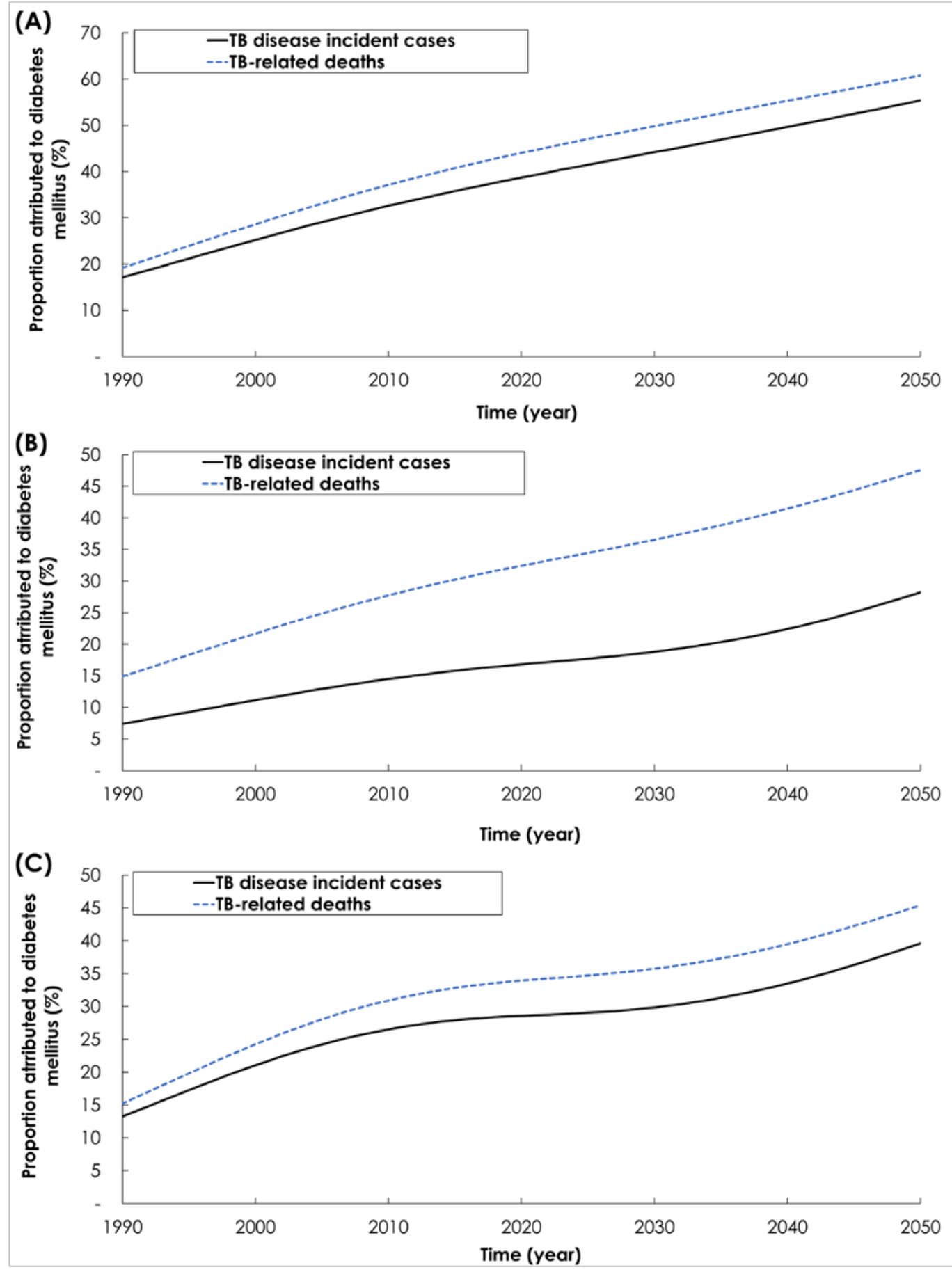


\section{Supplementary}

\section{Forecasting the Impact of Diabetes Mellitus on Tuberculosis Disease Incidence and Mortality in India}

Susanne F. Awad, ${ }^{1,2}$ Peijue Huangfu, ${ }^{2}$ Houssein H. Ayoub, ${ }^{1,3,4}$ Fiona Pearson, ${ }^{2}$ Soha R. Dargham, ${ }^{1}$ Julia Critchley, ${ }^{2}$ and Laith J. Abu-Raddad, ${ }^{1,4,5}$

${ }^{1}$ Infectious Disease Epidemiology Group, Weill Cornell Medicine-Qatar, Cornell University, Qatar Foundation - Education City, Doha, Qatar

${ }^{2}$ Population Health Research Institute, St George's, University of London, London, UK

${ }^{3}$ Department of Mathematics, Statistics, and Physics, Qatar University, Doha, Qatar

${ }^{4}$ Department of Healthcare Policy and Research, Weill Cornell Medicine, Cornell University, New York, New York, USA

${ }^{5}$ College of Health and Life Sciences, Hamad bin Khalifa University, Doha, Qatar 


\section{DETAILED DESCRIPTION OF THE TB-DM MATHEMATICAL MODEL}

We extended an earlier population-level deterministic mathematical model of the dynamics of tuberculosis-diabetes mellitus (TB-DM) interactions [1]. The model was described by sets of coupled nonlinear ordinary differential equations (Section 1.3) coded in MATLAB 2015a [2].

We stratified the population of India into compartments according to five-year age groups (indexed $a=1,2, \ldots, 20$ representing the 0 -99 age cohort), DM status, and TB progression states. We described TB natural history (for those with and without DM) by the progression states of susceptible, latent TB infection, TB disease, treated TB disease, and recovered (Figure S1).

Figure S1. A schematic diagram of the TB-DM model. The black and red lines indicate different TB natural histories depending on DM status. The blue box/line indicates the potential TB effect on DM.

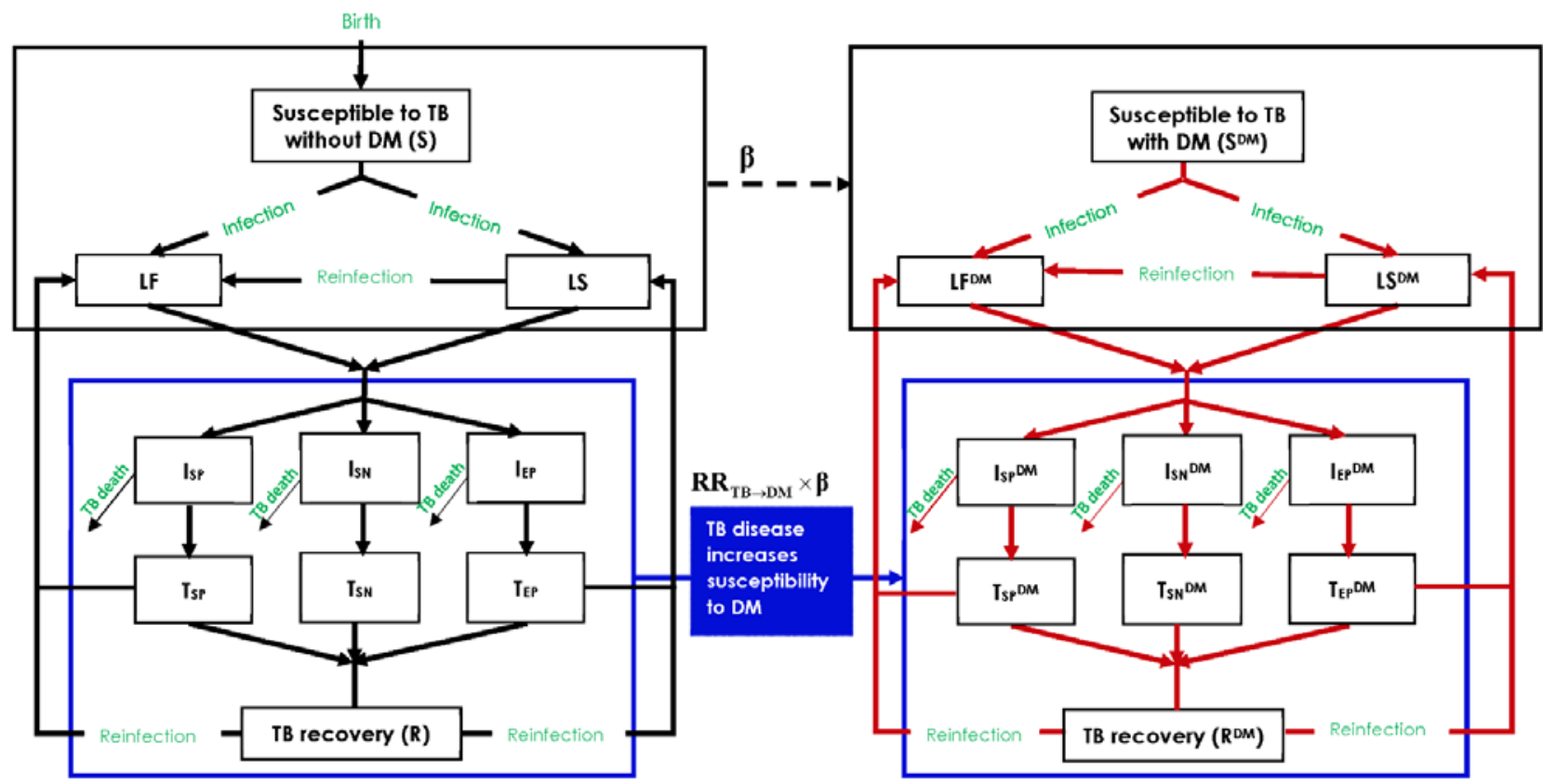

TB: tuberculosis; DM: diabetes mellitus; LF: latent-fast TB infection; LS: latent-slow TB infection; Isp: smear-positive pulmonary TB; ISN: smear-negative pulmonary TB; IEP: extra-pulmonary TB; Tsp: treated smear-positive pulmonary TB; TsN: treated smearnegative pulmonary TB; TEp: treated extra-pulmonary TB 


\subsection{TB transmission dynamics in absence of DM}

All individuals were born ( $\delta(a) \varphi(t) N_{\text {total }}$; here $\delta$ is equal one for $a=1$ and zero otherwise) susceptible to TB and DM ( $S$ ), and aged at a transition rate $\eta$ (i.e. from one age group to the next age group). In absence of DM, all individuals were at risk of developing DM at a rate $\beta(t, a)$ (except those $0-4$ years old; $a=1$ ), and at risk of natural mortality at a rate $\mu(t, a)$. TB susceptible individuals were at risk of TB infection at a rate $\lambda(t)$ (force of infection). A proportion $p$ and 1- $p$ of individuals move, upon TB infection, to the stage of TB latent fast progression ( $L^{F}$ ) and the stage of latent slow progression $\left(L^{S}\right)$, respectively. The proportion $p(a)$ differed between children $\left(<15\right.$ years old) and adults $\left(\geq 15\right.$ years old). Individuals in $L^{F}$ and $L^{S}$ were at risk of TB disease at a rate $\omega_{L F}$ and $\omega_{L S}$, respectively. Individuals in $L^{S}$ were also at risk of reinfection (at a rate $(1-q) \lambda$ ), but due to prior TB exposure and acquired immunity there was a proportional reduction $(q)$ in the susceptibility to TB infection.

TB disease individuals were characterized into the three states of smear-positive pulmonary ( $I^{S P}$ ), smear-negative pulmonary $\left(I^{S N}\right)$, and extra-pulmonary disease $\left(I^{E P}\right)$. The parameter $\alpha(a)$ identified the fraction of individuals going into each of these disease states, and differed between children and adults [3]. We considered individuals with the pulmonary TB disease types ( $I^{S P}$ and $I^{S N}$ ) infectious, but at varying levels. Individuals in the TB disease states could leave their state by TB-related mortality at a rate $\xi$, by spontaneous natural recovery at a rate $v$ (i.e. can recover without medical treatment), or by diagnosis and effective treatment at a rate $z \zeta$. Here, $\zeta$ is TB treatment rate, and $z$ is the proportion of new TB disease cases that successfully completed treatment (with or without) bacteriologic evidence of success ("cured" or "treatment 
completed"). This proportion was derived from seven treatment outcome measures given by the World Health Organization (WHO) [4]:

$z=\frac{\text { cure"+"treatment completed } "}{\text { cure"+ "treatment completed "+ "death"+ "treatment failure"+ default } "+\text { transferred } "+" \text { not evaluated }}$.

Treated individuals were characterized according to the three TB disease types: $T^{S P}, T^{S N}$, and $T^{E P}$. Individuals in the pulmonary treated states $\left(T^{S P}\right.$ and $\left.T^{S N}\right)$ were considered infectious, but at varying levels. Individuals in the treated states were assumed at risk of TB reinfection at a rate $(1-q) \lambda$, TB-related mortality at a rate $\xi$, spontaneous recovery at a rate $v$, or successful treatment completion at a rate $\psi$.

Individuals who are successfully treated, or those who spontaneously recover, enter the recovery stage ( $R$ ). Recovered TB individuals were assumed at risk of TB reinfection at a rate $(1-q) \lambda$.

The model accommodates (in principle) the risk of developing $\mathrm{DM}$ at a rate $R R_{T B \rightarrow D M} \times \beta$ among individuals with current or previous TB disease $\left(I^{S P}, I^{S N}, T^{S P}, T^{S N}\right.$, and $R$; Figure S1 blue line). Here, $R R_{T B \rightarrow D M}$ is the relative risk (RR) of developing $\mathrm{DM}$ in the population with a history of TB disease compared to the general population. However, given that our estimates were generated using a conservative approach, we opted not to factor this effect in our analysis since the evidence of this effect is still inconclusive.

\subsection{TB transmission dynamics in presence of DM}

Individuals with DM were assumed at higher risk of mortality due to DM complications at a rate $R R_{D M}(a) \mu(t, a)$. Here, $R R_{D M}$ is the RR of mortality in the DM population compared to the general population. 
Based on review of existing evidence, DM was assumed to affect 10 different stages of TB natural history and treatment outcomes, denoted as $E_{1}$ to $E_{10}$ in the equations in Section 1.3. The summary of the effects and their effect sizes is found in Table 1 of main manuscript and described in details in Section 3.1.

\subsection{Model structure}

TB transmission dynamics for the population without DM

TB and DM susceptible (i.e., TB susceptible individuals without DM):

$\frac{d S(a)}{d t}=\delta(a) \varphi(t) N_{\text {total }}+\eta[1-\delta(a)] S(a-1)-[\eta+\lambda(t)+\mu(t, a)+\beta(t, a)] S(a)$

$\underline{\text { TB latent infection: }}$

$$
\begin{aligned}
\frac{d L^{F}(a)}{d t}= & \eta[1-\delta(a)] L^{F}(a-1)+p(a) \lambda(t) S(a)+p(a)[1-q] \lambda(t) L^{S}(a)+p(a)[1-q] \lambda(t)\left[R(a)+T^{S P}(a)+T^{S N}(a)+T^{E P}(a)\right] \\
& -\left[\eta+\omega_{L F}+\mu(t, a)+\beta(t, a)\right] L^{F}(a) \\
\frac{d L^{S}(a)}{d t}= & \eta[1-\delta(a)] L^{S}(a-1)+[1-p(a)] \lambda(t) S(a)+[1-p(a)][1-q] \lambda(t)\left[R(a)+T^{S P}(a)+T^{S N}(a)+T^{E P}(a)\right] \\
& -\left[\eta+p(a)[1-q] \lambda(t)+\omega_{L S}+\mu(t, a)+\beta(t, a)\right] L^{S}(a)
\end{aligned}
$$

TB disease:

$$
\begin{aligned}
& \frac{d I^{S P}(a)}{d t}=\eta[1-\delta(a)] I^{S P}(a-1)+\alpha^{L F F S P P}(a) \omega_{L F} L^{F}(a)+\alpha^{L S O S P P}(a) \omega_{L S} L^{S}(a)-\left[\eta+\xi_{S P}+z \zeta_{S P}+v_{S P}+\mu(t, a)+R R_{T B \rightarrow D M} \beta(t, a)\right] I^{S P}(a) \\
& \frac{d I^{S N}(a)}{d t}=\eta[1-\delta(a)] I^{S N}(a-1)+\alpha^{L F F S S N}(a) \omega_{L F} L^{F}(a)+\alpha^{L S O S N}(a) \omega_{L S} L^{S}(a)-\left[\eta+\xi_{S N}+z \zeta_{S N}+v_{S N}+\mu(t, a)+R R_{T B \rightarrow D M} \beta(t, a)\right] I^{S N}(a) \\
& \frac{d I^{E P}(a)}{d t}=\eta[1-\delta(a)] I^{E P}(a-1)+\alpha^{L F F O P P}(a) \omega_{L F} L^{F}(a)+\alpha^{L S O E P P}(a) \omega_{L S} L^{S}(a)-\left[\eta+\xi_{E P}+z \zeta_{E P}+v_{E P}+\mu(t, a)+R R_{T B \rightarrow D M} \beta(t, a)\right] I^{E P}(a)
\end{aligned}
$$




\section{Treated TB disease:}

$$
\begin{aligned}
& \frac{d T^{S P}(a)}{d t}=\eta[1-\delta(a)] T^{S P}(a-1)+z \zeta_{S P} I^{S P}(a)-\left[\eta+v_{T S P}+\psi_{T S P}+(1-q) \lambda(t)+\xi_{T S P}+\mu(t, a)+R R_{T B \rightarrow D M} \beta(t, a)\right] T^{S P}(a) \\
& \frac{d T^{S N}(a)}{d t}=\eta[1-\delta(a)] T^{S N}(a-1)+z \zeta_{S N} I^{S N}(a)-\left[\eta+v_{T S N}+\psi_{T S N}+(1-q) \lambda(t)+\xi_{T S N}+\mu(t, a)+R R_{T B \rightarrow D M} \beta(t, a)\right] T^{S N}(a) \\
& \frac{d T^{E P}(a)}{d t}=\eta[1-\delta(a)] T^{E P}(a-1)+z \zeta_{E P} I^{E P}(a)-\left[\eta+v_{T E P}+\psi_{T E P}+(1-q) \lambda(t)+\xi_{T E P}+\mu(t, a)+R R_{T B \rightarrow D M} \beta(t, a)\right] T^{E P}(a)
\end{aligned}
$$

\section{$\underline{\text { Recovered: }}$}

$$
\begin{aligned}
\frac{d R(a)}{d t} & =\eta[1-\delta(a)] R(a-1)+\underbrace{\psi_{T S P} T^{S P}(a)+\psi_{T S N} T^{S N}(a)+\psi_{T E P} T^{E P}(a)}_{\text {Treatment success }} \\
& +\underbrace{v_{S P} I^{S P}(a)+v_{S N} I^{S N}(a)+v_{E P} I^{E P}(a)+v_{T S P} T^{S P}(a)+v_{T S N} T^{S N}(a)+v_{T N P} T^{E P}(a)}_{\text {Spontaneous recovery }} \\
& -[\eta+[1-q] \lambda(t)+\mu(t, a)+\beta(t, a)] R(a)
\end{aligned}
$$

TB transmission dynamics for the population with DM (aged $\geq 5$ years)

TB susceptible with DM:

$\frac{d S^{D M}(a)}{d t}=\eta[1-\delta(a)] S^{D M}(a-1)+\beta(t, a) S(a)-\left[\eta+E_{1} \lambda(t)+R R_{D M}(a) \mu(t, a)\right] S^{D M}(a)$

$\underline{\text { TB latent infection with DM: }}$

$$
\begin{aligned}
& \frac{d L^{F_{\omega \prime}}(a)}{d t}=\eta[1-\delta(a)] L^{F_{\omega \mu}}(a-1)+\beta(t, a) L^{F}(a)+E_{2} p(a) E_{1} \lambda(t) S^{D M}(a)+p(a) E_{4}[1-q] \lambda(t) L^{S_{\alpha u}}(a)
\end{aligned}
$$

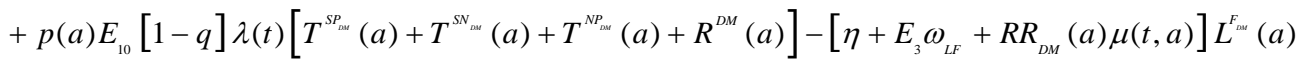

$$
\begin{aligned}
& \frac{d L^{S_{o u}}(a)}{d t}=\eta[1-\delta(a)] L^{S_{\text {oum }}}(a-1)+\beta(t, a) L^{S}(a)+\left[1-E_{2} p(a)\right] E_{1} \lambda(t) S^{D M}(a)
\end{aligned}
$$

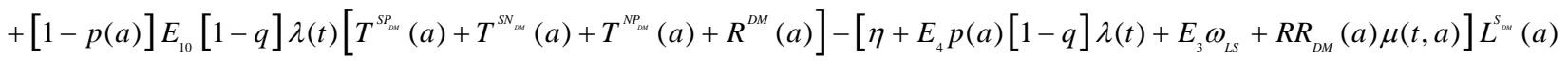

\section{TB disease with DM:}




$$
\begin{aligned}
& \frac{d I^{S P_{D M}}(a)}{d t}=\eta[1-\delta(a)] I^{S P_{D M}}(a-1)+R R_{T B \rightarrow D M} \beta(t, a) I^{S P}(a)+\alpha^{\left(L F F S P P_{D M}\right.}(a) E_{3} \omega_{L F} L^{F_{D M}}(a)+\alpha^{\left(L S T O S P P_{D M}\right.}(a) E_{3} \omega_{L S} L^{S_{D M}}(a) \\
& -\left[\eta+E_{8} z \zeta_{S P}+E_{9} v_{S P}+E_{7} \xi_{S P}+R R_{D M}(a) \mu(t, a)\right] I^{S P_{D M}}(a) \\
& \frac{d I^{S N_{D M}}(a)}{d t}=\eta[1-\delta(a)] I^{S N_{D M}}(a-1)+R R_{T B \rightarrow D M} \beta(t, a) I^{S N}(a)+\alpha^{(L F T S S)_{D M}}(a) E_{3} \omega_{L F} L^{F_{D M}}(a)+\alpha^{(L S T O S N)_{D M}}(a) E_{3} \omega_{L S} L^{S_{D M}}(a) \\
& -\left[\eta+E_{8} z \zeta_{S N}+E_{9} \nu_{S N}+E_{7} \xi_{S N}+R R_{D M}(a) \mu(t, a)\right] I^{S N_{D M}}(a) \\
& \frac{d I^{E P_{D M}}(a)}{d t}=\eta[1-\delta(a)] I^{E P_{D M}}(a-1)+R R_{T B \rightarrow D M} \beta(t, a) I^{E P}(a)+\alpha^{\left(L F \leftarrow \circ P_{D M}\right.}(a) E_{3} \omega_{L F} L_{a}^{F_{D M}}(a)+\alpha^{\left(L S T O E P P_{D M}\right.}(a) E_{3} \omega_{L S} L^{S_{D M}}(a) \\
& -\left[\eta+E_{8} Z \zeta_{E P}+E_{9} \nu_{E P}+E_{7} \xi_{E P}+R R_{D M}(a) \mu(t, a)\right] I^{E P_{D M}}(a)
\end{aligned}
$$

Treated TB disease with DM:

$$
\begin{aligned}
\frac{d T^{S P_{D M}}(a)}{d t}=\eta[1-\delta(a)] T^{S P_{D M}}(a-1)+R R_{T B \rightarrow D M} \beta(t, a) T^{S P}(a)+E_{8} Z \zeta_{S P} I^{S P_{D M}}(a) \\
-\left[\eta+E_{9} v_{T S P}+E_{9} \psi_{T S P}+E_{10}[1-q] \lambda(t)+E_{7} \xi_{T S P}+R R_{D M}(a) \mu(t, a)\right] T^{S P_{D M}}(a) \\
\frac{d T^{S N_{D M}}(a)}{d t}=\eta[1-\delta(a)] T^{S N_{D M}}(a-1)+R R_{T B \rightarrow D M} \beta(t, a) T^{S N}(a)+E_{8} Z \zeta_{S N} I^{S N_{D M}}(a) \\
-\left[\eta+E_{9} v_{T S N}+E_{9} \psi_{T S N}+E_{10}[1-q] \lambda(t)+E_{7} \xi_{T S N}+R R_{D M}(a) \mu(t, a)\right] T^{S N_{D M}}(a) \\
\frac{d T^{E P_{D M}}(a)}{d t}=\eta[1-\delta(a)] T^{E P_{D M}}(a-1)+R R_{T B \rightarrow D M} \beta(t, a) T^{E P}(a)+E_{8} Z \zeta_{E P} I^{E P_{D M}}(a) \\
-\left[\eta+E_{9} v_{T E P}+E_{9} \psi_{T E P}+E_{10}[1-q] \lambda(t)+E_{7} \xi_{T E P}+R R_{D M}(a) \mu(t, a)\right] T^{E P_{D M}}(a)
\end{aligned}
$$

$\underline{\text { TB recovered with DM: }}$

$$
\begin{aligned}
& \frac{d R^{D M}(a)}{d t}=\eta R^{D M}(a-1)+R R_{T B \rightarrow D M} \beta(t, a) R(a)+\underbrace{E_{9}\left[\psi_{T S P} T^{S P_{D M}}(a)+\psi_{T S N} T^{S N_{D M}}(a)+\psi_{T E P} T^{E P_{D M}}(a)\right]}_{\text {Treatmentsuccess }} \\
& \quad+\underbrace{E_{9}\left[v_{S P} I^{S P_{D M}}(a)+v_{S N} I^{S N_{D M}}(a)+v_{E P} I^{E P_{D M}}(a)+v_{T S P} T^{S P_{D M}}(a)+v_{T S N} T^{S N_{D M}}(a)+v_{T E P} T^{E P_{D M}}(a)\right]}_{\text {Spontaneous recovery }} \\
& \quad-\left[\eta+E_{10}[1-q] \lambda(t)+R R_{D M}(a) \mu(t, a)\right] R^{D M}(a)
\end{aligned}
$$

Here, the parameter $\alpha$ for the population with DM was determined according to:

$$
\begin{aligned}
& \alpha^{(L F T S P)_{D M}}(a)=\alpha^{(L S T O S P)_{D M}}(a)=E_{5}^{S P}(a) \alpha^{L F F S P}(a), \\
& \alpha^{(L F T O S N)_{D M}}(a)=\alpha^{(L S T L S N)_{D M}}(a)=E_{5}^{S N}(a) \alpha^{L F t o S N}(a), \\
& \alpha^{\left(L F F O E P P_{D M}\right.}(a)=\alpha^{\left(L S G O E P P_{D M}\right.}(a)=\alpha^{L F F \leftarrow O P P}(a)
\end{aligned}
$$


while,

$$
\begin{aligned}
& E_{5}^{S P}(a)=\kappa \frac{\alpha^{(L F t o S P)}(a)+\alpha^{(L F t o S N)}(a)}{\left[\kappa \alpha^{(L F t o S P)}(a)+\kappa^{\prime} \alpha^{(L F t o S N)}(a)\right]}, \\
& E_{5}^{S N}(a)=\kappa^{\prime} \frac{\alpha^{(L F t o S P)}(a)+\alpha^{(L F t o S N)}(a)}{\left[\kappa \alpha^{(L F t o S P)}(a)+\kappa^{\prime} \alpha^{(L F t o S N)}(a)\right]}
\end{aligned}
$$

and

$$
\begin{aligned}
& \kappa=\frac{P_{D M}^{S P}}{P_{N D M}^{S P}} \\
& \kappa^{\prime}=\frac{1-P_{D M}^{S P}}{1-P_{N D M}^{S P}}
\end{aligned}
$$

Here, $P_{D M}^{S P}$ and $P_{N D M}^{S P}$ are the proportions of smear-positive pulmonary TB cases in the DM and non-DM groups, respectively. These proportions were obtained from observational studies discussed in Section 3.1.

\subsection{Demographic parameters}

Total number of individuals in the population, $N_{\text {total }}$, was given by:

$$
N_{\text {total }}=\sum_{a=1}^{20}\left(\begin{array}{l}
S(a)+L^{F}(a)+L^{S}(a)+I^{S P}(a)+I^{S N}(a)+I^{E P}(a)+T^{S P}(a)+T^{S N}(a)+T^{E P}(a)+R(a) \\
+S^{D M}(a)+L^{F_{D M}}(a)+L^{S_{D M}}(a)+I^{S P_{D M}}(a)+I^{S N_{D M}}(a)+I^{E P_{D M}}(a)+T^{S P_{D M}}(a)+T^{S N_{D M}}(a)+T^{E P_{D M}}(a)+R^{D M}(a)
\end{array}\right)
$$

The population growth rate $(\varphi(t))$ and the natural mortality rate $(\mu(t, a))$ were described by the following functions, providing a robust fit of population growth and age structure in India:

$\varphi(t)=a_{0} e^{-\left(\frac{t-t_{0}}{b_{0}}\right)^{2}}$

and 
$\mu(t, a)=\frac{a_{1} e^{-\left(\frac{t-t_{1}}{b_{1}}\right)^{2}}}{\left[1+e^{-b_{2}\left(a-a_{2}\right)}\right]}$

Here the parameters $a_{0}, a_{1}, a_{2}, t_{0}, t_{1}, b_{0}, b_{1}$, and $b_{2}$ were obtained by fitting the model to India's demographic data from the database of the Population Division of the United Nations Department of Economic and Social Affairs [5].

\subsection{TB force of infection and temporal evolution of TB contact rate}

Assuming that the mixing between individuals in the population was random, the TB force of infection $(\lambda)$ was determined by the probability of transmission per respiratory contact $(u)$, the respiratory contact rate within a population $(\varepsilon)$, the effect of DM on TB infectiousness $\left(E_{6}\right)$, and the relative infectiousness of individuals with each type of TB disease (whether untreated or treated) compared to the infectiousness of individuals with smear-positive pulmonary TB $(h)$ :

$$
\begin{aligned}
\lambda(t)= & \frac{u \varepsilon(t) \sum_{a=1}^{20}\left(h_{S P} I^{S P}(t, a)+h_{S N} I^{S N}(t, a)+h_{E P} I^{E P}(t, a)+h_{T S P} T^{S P}(t, a)+h_{T S N} T^{S N}(t, a)+h_{T E P} T^{E P}(t, a)\right)}{N_{\text {tout }}(t, a)} \\
& +\frac{E_{6} u \varepsilon(t) \sum_{a=1}^{20}\left(h_{S P_{D M}} I^{S P_{D M}}(t, a)+h_{S N_{D M}} I^{S N_{D M}}(t, a)+h_{E P_{D M}} I^{E P_{D M}}(t, a)+h_{T S P_{D M}} T^{S P_{D M}}(t, a)+h_{T S N_{D M}} T^{S N_{D M}}(t, a)+h_{T E P_{D M}} T^{E P_{D M}}(t, a)\right)}{N_{\text {tootl }}(t, a)}
\end{aligned}
$$

Given the evidence for declining TB incidence in India, a temporal change in $\varepsilon$ was incorporated in the model. The temporal variation was characterized through a Wood-Saxon function [6,7]. This function is mathematically designed to describe and characterize transitions. It parameterizes a transition in terms of its scale or strength, smoothness or abruptness, duration, and the turning year [6,7]. Using the Wood-Saxon parameterization, $\varepsilon(t)$ was given by: 
$\varepsilon(t)=\varepsilon_{0}\left(1+\frac{Z}{1+\exp \left[\left(t-\xi_{\text {Turning }}\right) / \xi_{\text {Duration }}\right]}\right)$

Here, $\varepsilon_{0}$ is the asymptotic value that describes the contact rate well after the transition, $\mathrm{Z}$ is the level of change in $\varepsilon(t)$ during the transition from $\varepsilon_{0}(1+Z)$ before the transition to $\varepsilon_{0}$ after the transition, $\xi_{\text {Duration }}$ describes the transition duration parameter, and $\xi_{\text {Turning }}$ is the turning point year at which the contact rate crosses half way towards its asymptotic value of $\varepsilon_{0}$. The parameters $\varepsilon_{0}, \mathrm{Z}, \xi_{\text {Duration }}$, and $\xi_{\text {Turning }}$ were obtained by fitting the model to available empirical data on TB-incidence and mortality from the WHO’s Global Health Observatory data repository [8].

\subsection{TB treatment rate and temporal evolution of TB case detection rate}

Treatment rate in the model depended on TB disease type and was determined using the case detection rates $\left(C_{\text {DetSP }}, C_{\text {DetSN }}\right.$, and $\left.C_{\text {DetEP }}\right)$, TB-related mortality rates $\left(v_{S P}, v_{S N}, v_{E P}\right)$, and spontaneous recovery rates $\left(\xi_{S P}, \xi_{S N}, \xi_{E P}\right)$ :

$$
\begin{aligned}
& \zeta_{S P}=C_{D e t S P}\left(v_{S P}+\xi_{S P}\right) /\left(1-C_{D e t S P}\right) \\
& \zeta_{S N}=C_{D e t S N}\left(v_{S N}+\xi_{S N}\right) /\left(1-C_{D e t S N}\right) \\
& \zeta_{E P}=C_{D e t E P}\left(v_{E P}+\xi_{E P}\right) /\left(1-C_{D e t E P}\right)
\end{aligned}
$$

Given the evidence for increasing TB case detection in India [9], temporal changes in TB case detection rates were incorporated in the model. Moreover, given the likelihood of underreporting of treatment among TB cases, TB case detection rate for India was derived by fitting the model to TB incidence rate and mortality rate. The temporal variation was parametrized through a logistic function: 
$C_{\text {DetX }}(t)=\frac{p_{1}}{\left[1+e^{-p_{2}\left(t-p_{3}\right)}\right]}$.

Here, the parameters $p_{1}, p_{2}$, and $p_{3}$ were obtained by fitting the model to available empirical data on TB-incidence and mortality rates from the WHO’s Global Health Observatory data repository [8].

\subsection{DM incidence rate}

Given the evidence for increasing DM incidence and prevalence in India [10], the DM incidence rate in the TB-DM model was assumed to be time and age dependent, and was parameterized through a combined Gaussian-logistic function:

$\beta(t, a)=\frac{c_{1} e^{-\left(\frac{t-t_{1}}{d_{1}}\right)^{2}}}{\left[1+e^{-d_{2}\left(a-c_{2}\right)}\right]}$.

Here, $c_{1}, c_{2}, t_{1}, d_{1}$, and $d_{2}$ are fitting parameters obtained by fitting the TB-DM model to the time series of DM prevalence in India as provided through the International Diabetes Federation [10]. The shape of the age-distribution of DM prevalence was based on the national Indian Council of Medical Research-India Diabetes study [11]. 


\section{POPULATION ATTRIBUTABLE FRACTION}

The epidemiologic implications of the TB-DM interactions in India were assessed using two (incidence and mortality) “true” population attributable fraction ( $P A F)$ measures, representing the proportions of all TB disease incidence or mortality that could be prevented if there was no interaction between TB and DM. They were defined as:

$P A F_{\text {True }}^{D}=\frac{D_{T B-D M}-D_{\text {Counter-factual }}}{D_{T B-D M}}$.

Here, $D$ indicates the epidemiological measure of incidence or mortality. $D_{T B-D M}$ is the measure in a scenario where there is a biological synergy between TB and DM, while $D_{\text {Counter-factual }}$ is the measure in a counter-factual scenario where the biological synergy between TB and DM is absent. 


\section{DATA SOURCES}

The TB-DM interaction model was parameterized using empirical epidemiological and natural

history data from multiple sources.

\section{1. $\quad$ TB epidemiological and natural history data}

The model's parameter values for TB natural history in absence of DM, along with their references, are listed in Table S1.

Table S1. Model assumptions in terms of parameter values.

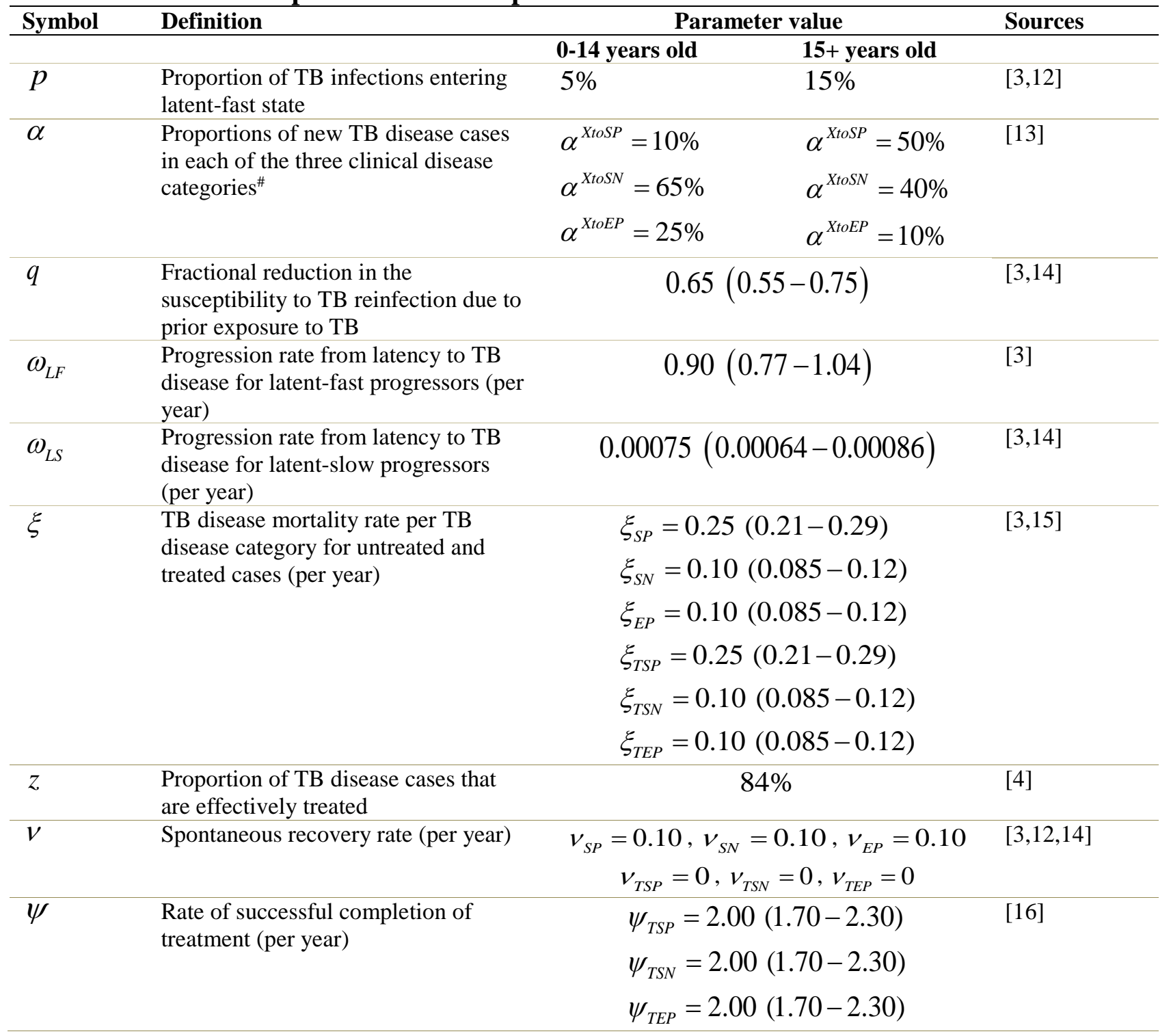




\begin{tabular}{|c|c|c|c|c|}
\hline$u$ & $\begin{array}{l}\text { Transmission probability per } \\
\text { respiratory contact }\end{array}$ & & $\%$ & [3] \\
\hline$h$ & $\begin{array}{l}\text { Relative infectiousness for each of the } \\
\text { three disease categories and treatment } \\
\text { categories with respect to smear- } \\
\text { positive pulmonary disease }\end{array}$ & $h$ & $\begin{array}{l}100 \% \\
25 \% \\
0 \% \\
13 \% \\
=3.3 \% \\
0 \%\end{array}$ & {$[3,17,18]$} \\
\hline$R R_{D M}^{\text {Age-group }}$ & $\begin{array}{l}\text { Relative risk of mortality in people } \\
\text { with DM (per age group) compared to } \\
\text { the general population }\end{array}$ & $\begin{array}{l}R R_{D M}^{0-4}=1.00 \\
R R_{D M}^{5-14}=2.67\end{array}$ & $\begin{array}{l}R R_{D M}^{15-29}=4.83 \\
R R_{D M}^{30-39}=4.46 \\
R R_{D M}^{40-49}=2.67 \\
R R_{D M}^{50-59}=2.19 \\
R R_{D M}^{60-69}=1.85 \\
R R_{D M}^{70-79^{+}}=1.59\end{array}$ & $\begin{array}{l}\text { Calculated } \\
\text { based on } \\
{[19,20]}\end{array}$ \\
\hline
\end{tabular}

\begin{tabular}{llll}
\hline Country specific variables & & \\
\hline$N$ & Total population & $\begin{array}{l}\text { For each year per the database of the } \\
\text { Population Division of the United Nations } \\
\text { Department of Economic and Social Affairs }\end{array}$ & {$[5]$} \\
\hline$\varphi$ & Birth rate & Gaussian function & $\begin{array}{l}\text { Fitting } \\
\text { parameters }\end{array}$ \\
\hline$\mu$ & Natural mortality rate & $\begin{array}{l}\text { Combination of logistic and Gaussian } \\
\text { functions }\end{array}$ & $\begin{array}{l}\text { Fitting } \\
\text { parameters }\end{array}$ \\
\hline$C$ & $\begin{array}{l}\text { Case detection rate per TB disease } \\
\text { category }\end{array}$ & Logistic function & $\begin{array}{l}\text { Fitting } \\
\text { parameters }\end{array}$ \\
\hline$\varepsilon$ & Respiratory contact rate (per year) & Wood Saxon (logistic function) & $\begin{array}{l}\text { Fitting } \\
\text { parameters }\end{array}$ \\
\hline$\beta$ & DM incidence rate (per year) & $\begin{array}{l}\text { Combination of logistic and Gaussian } \\
\text { functions }\end{array}$ & $\begin{array}{l}\text { Fitting } \\
\text { parameters }\end{array}$ \\
\hline
\end{tabular}

\#The three clinical categories are smear-positive pulmonary (SP), smear-negative pulmonary (SN), and extrapulmonary (EP) tuberculosis. $X$ is latent slow (LS) or latent fast (LF).

\subsection{Parametrization of DM-on-TB effects}

We incorporated seven out of ten potential DM effects on TB's natural history and treatment outcomes. These are, along with their parameter values, summarized in Table 1 of main text. A brief justification and summary of the evidence for each parameter can be found below. Further details can be found in reference [1].

Effect 1-Susceptibility: DM increases susceptibility to TB infection 
Supported by existing evidence [21,22], and based on a recent population-based cross sectional study using the 2011-12 National Health and Nutrition Examination Survey (NHANES) data [23], a 1.5 (95\% confidence interval (CI): 1.0-2.2) increased risk of TB infection for individuals with DM, compared to individuals without DM, was incorporated in the model.

Effect 2-Fast progression: DM increases the proportion of TB infections entering latent-fast state as opposed to latent-slow state

A recent meta-analysis of all available study designs $(\mathrm{N}=44)$ and four prospective cohort studies found that DM was associated with a $2.00-$ fold (95\% CI: 1.78-2.24) and a 3.59-fold (95\% CI: 2.25-5.73) increased risk of TB disease, respectively.[24] However, it is not possible to determine from these studies whether DM increases $i$ ) the proportion of TB infections entering latent-fast state as opposed to latent-slow state (Effect 2-Fast progression), or ii) reactivation of latent slow TB cases (Effect 3-Reactivation), or iii) reinfection of latent slow TB cases (Effect 4Reinfection), or iv) a combination of the three effects.

Given that these potential effects could be acting simultaneously, and their individual effects cannot be disentangled from each other [1], we included in the model only one of these effects (Effect 2-Fast progression). The exact effect size for Effect 2-Fast progression was estimated by fitting the model outcome to the more conservative pooled association of 2.00 (based on all studies) [24]. The model was also fitted to the pooled hazard ratio of 3.59 (based only on the prospective cohort studies) [24] in a sensitivity analysis. No effect sizes were assumed for Effect 3-Reactivation and Effect 4-Reinfection, as the impacts of these on TB natural history is presumably implicitly captured by Effect 2-Fast progression.

Only two studies estimated the age-specific relative risks (RRs) of the effect of DM on TB disease, and these demonstrated a decrease in the RR with age [25,26]. Among DM individuals, 
the RR of TB disease was estimated at 7.79 among those aged 20-29, 9.98 among those aged 30-39, 4.72 among those aged 40-49, 2.30 among those aged 50-59, and 1.76 among those aged $60+$ [25]. The age-specific RRs of the effect of DM on TB disease [25] were incorporated in the model (as opposed to the overall effect regardless of age), but only in sensitivity analysis. When this was done, the age effects reported in reference [25] were scaled down to reach the two-fold overall RR of the effect of DM on TB disease in the total population (as determined in the metaanalysis based on all studies [24]).

Effect 5-Smear positivity: DM increases the proportion of new pulmonary TB disease cases going to smear-positive as opposed to smear-negative

Based on current evidence $[27,28]$, the proportion of individuals with extra-pulmonary TB as opposed to pulmonary (PTB) in the model was assumed not to differ based on DM status. However, studies have demonstrated that individuals with concurrent PTB and DM were more likely to be sputum acid-fast bacilli (AFB) smear-positive (i.e. have SP-PTB) as opposed to smear-negative (SN-PTB) [29-44]. Based on this evidence, the fraction of individuals who develop SP-PTB as opposed to SN-PTB was assumed to differ by DM status. These fractions were estimated using the pooled mean proportions (out of all PTB cases) by DM status, with the pooling done using a DerSimonian-Laird random-effects model [45] (Figure S2). 
Figure S2. Forest plots presenting the outcomes of the pooled mean proportion of smearpositive pulmonary tuberculosis in different populations (A) without diabetes mellitus and (B) with concurrent diabetes mellitus.

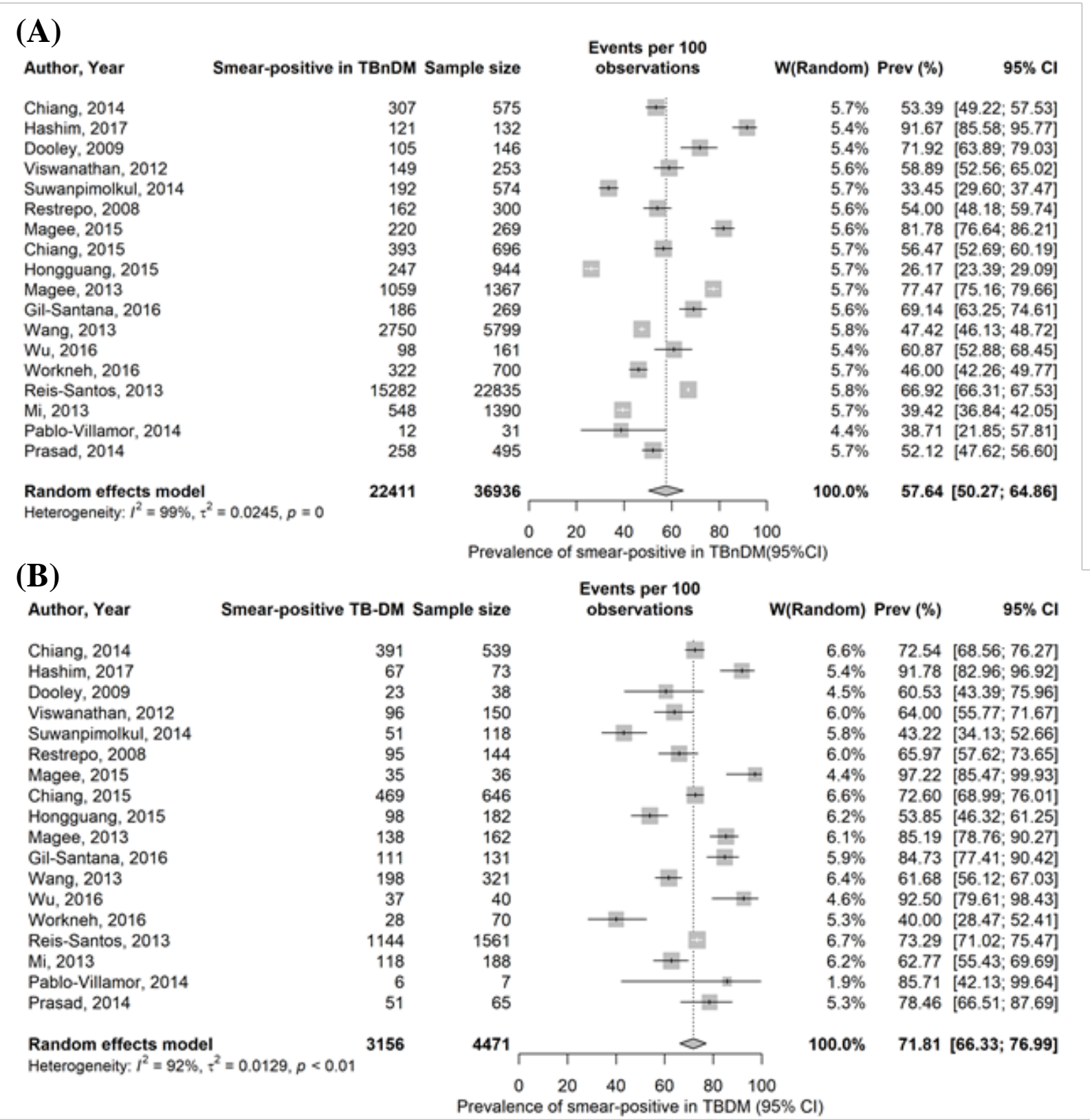

Accordingly using Equations S1-S3 in Section 1.3, for adults upon progressing to TB disease from both $L^{S}$ and $L^{F}, 63.1 \%$ of those with DM will develop SP-PTB compared to 50.0\% of those without DM; $26.9 \%$ of those with DM will develop SN-PTB compared to $40.0 \%$ of those without DM. For children, upon progressing to TB disease from both $L^{S}$ and $L^{F}, 16.8 \%$ of those 
with DM will develop SP-PTB compared to $10.0 \%$ of those without DM; $58.2 \%$ of those with DM will be develop SN-PTB compared to $65.0 \%$ of those without DM. The proportions without DM (for adults and children) were based on earlier work [3].

Effect 6-Disease infectiousness: DM increases the infectiousness of PTB (SP-PTB and SN-PTB) for untreated and treated TB disease cases

DM was found to be an independent risk factor associated with increased M. tuberculosis bacterial load (based on AFB sputum smear examination) [18,28,29,31,46-48]. Based on this evidence, this effect was incorporated in the model by increasing TB infectiousness among those with concurrent PTB and DM. First, TB infectiousness was assumed to be linearly proportional to M. tuberculosis bacterial load. Second, the ratio of TB bacterial load between concurrent TB and DM and TB with no DM individuals was assumed to be equal to the ratio of infectiousness between concurrent TB and DM and TB with no DM individuals. We used available studies to estimate a weighted average of the ratio of bacterial load between concurrent TB and DM and TB with no DM. Accordingly, infectiousness of TB among those with DM was assumed to increase by 1.46-fold compared to those without DM.

Effect 7-TB mortality: DM increases the hazard of TB-related mortality for TB disease cases

Evidence supports an association between DM and TB-related mortality [27,31,49-53]. A recent systematic review and meta-analysis estimated a pooled mean crude odds ratio (OR) of 2.11 (95\% CI: 1.76-2.51) across 48 studies, and an adjusted pooled mean OR of 4.95 (95\% CI: 2.699.10) across four studies that appropriately adjusted for confounders [53]. Based on this evidence, an effect size of 2.11 was incorporated in the TB-DM model as part of our conservative approach for estimating the impact of DM on TB, and an effect size of 4.95 was incorporated as part of a sensitivity analysis. We assumed that, among those who have TB 
disease, DM affected (relatively) TB mortality rate similarly for those treated and untreated for TB. The OR of 2.11 was converted to a hazard ratio (HR) using the following equation for Effect 7-TB mortality for untreated TB-DM cases:

$$
\mathrm{E}_{7}^{X}=\frac{\mathrm{OR}\left(E_{8} z \zeta_{X}+E_{9}^{X} v_{X}\right)}{z \zeta_{X}+v_{X}}
$$

Here, $X$ is $S P, S N$, or $E P$, while $E_{8}$ and $E_{9}$ are the effects of DM on TB cure and recovery, respectively (see below). The OR was also converted to a HR using the following equation for Effect 7-TB mortality for treated TB-DM cases:

$$
\mathrm{E}_{7}^{X}=\frac{\mathrm{OR}\left[E_{9}^{X}\left(v_{X}+\psi_{X}\right)+E_{10}(1-q) \lambda\right]}{v_{X}+\psi_{X}+(1-q) \lambda} .
$$

Here, $X$ is TSP, TSN, or TEP, while $E_{10}$ is the effect of DM on TB reinfection after recovery (see below).

Effect 8-Treatment failure: DM reduces the proportion of successful treatment

Several studies reported on "cure" and "treatment completed" as well as the other treatment outcome measures to calculate the proportion of successful treatment with and without DM [42,47,54-67]. Pooling the RR across these studies using a DerSimonian-Laird random-effects model [45], the RR for treatment success was 0.96 (95\% CI: 0.93-1.00; Figure S3). Because the RR was not statistically significant, this effect was not included in the TB-DM model—-the RR for Effect 8-Treatment failure was assumed to equal 1. 
Figure S3. Forest plot presenting the outcome of the pooled mean crude relative risk of tuberculosis treatment success with or with no DM in different populations.

\begin{tabular}{|c|c|c|c|c|c|c|c|}
\hline \multirow{2}{*}{$\begin{array}{l}\text { Author, Year } \\
\text { Ambrosetti, } 1999\end{array}$} & \multicolumn{2}{|c|}{$\begin{array}{c}D M \\
\text { TB curedTB not cured }\end{array}$} & \multicolumn{2}{|c|}{$\begin{array}{c}\text { non-DM } \\
\text { TB curedTB not cure }\end{array}$} & & \multicolumn{2}{|c|}{ Relative Risk [ $95 \% \mathrm{Cl}]$} \\
\hline & 22 & 10 & 601 & 136 & $1=$ & & $0.84[0.67,1.07]$ \\
\hline Ambrosetti, 1999 & 36 & 14 & 656 & 117 & $\sin$ & & $0.85[0.71,1.01]$ \\
\hline Ambrosetti, 1999 & 33 & 7 & 519 & 148 & -1 & & $1.06[0.91,1.23]$ \\
\hline Centis, 2000 & 26 & 15 & 693 & 366 & 㟧 & & $0.97[0.76,1.23]$ \\
\hline Centis, 2002 & 28 & 12 & 649 & 203 & $1+-1$ & & $0.92[0.75,1.13]$ \\
\hline Mboussa, 2003 & 17 & 23 & 77 & 15 & $\longmapsto$ & & $0.51[0.35,0.74]$ \\
\hline Singla, 2006 & 130 & 7 & 367 & 16 & $\varphi$ & & $0.99[0.95,1.03]$ \\
\hline Tatar, 2009 & 70 & 3 & 72 & 6 & 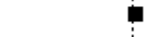 & & $1.04[0.96,1.13]$ \\
\hline Sangral, 2012 & 19 & 4 & 213 & 46 & -1 & & $1.00[0.83,1.22]$ \\
\hline Jiménez-Corona, 2013 & 308 & 55 & 711 & 136 & ب & & $1.01[0.96,1.07]$ \\
\hline Sulaiman, 2013 & 258 & 84 & 717 & 197 & 4 & & $0.96[0.90,1.03]$ \\
\hline K V N., 2013 & 554 & 113 & 1839 & 287 & $\dot{\varphi}$ & & $0.96[0.92,1.00]$ \\
\hline Reis-Santos, 2013 & 441 & 262 & 12826 & 4221 & a: & & $0.83[0.79,0.88]$ \\
\hline Viswanathan, 2014 & 80 & 9 & 115 & 5 & + & & $0.94[0.87,1.02]$ \\
\hline Alo, 2014 & 47 & 6 & 272 & 60 & - & & $1.08[0.97,1.21]$ \\
\hline Viswanathan, 2014 & 91 & 5 & 142 & 7 & $\dot{\varphi}$ & & $0.99[0.94,1.05]$ \\
\hline Cavanaugh, 2015 & 93 & 8 & 162 & 12 & $\dot{\varphi}$ & & $0.99[0.92,1.06]$ \\
\hline \multirow[t]{3}{*}{ Random effect model } & & & & & 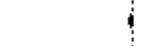 & & $0.96[0.93,1.00]$ \\
\hline & & & & Г & i & & \\
\hline & & & & 0.05 & 0.25 & 4 & \\
\hline
\end{tabular}

Effect 9-Recovery: DM reduces the rate of TB recovery (prolonging the recovery time) for those who recover naturally or due to treatment

Three studies reported on and compared the number of days it takes to convert from smear positive to smear negative among treated concurrent TB-DM cases and TB-non-DM cases [18,33,61]. The average ratio of the inverse duration (with DM compared to no DM) was pooled across studies by weighting by sample size. The pooled inverse duration ratio was 0.82 implying that DM reduces TB recovery rates ( $\psi$ and $v$ ) by $18 \%$.

Effect 10-Cured reinfection: DM increases susceptibility to TB reinfection among those treated or recovered from TB disease

TB reinfection was defined as a subsequent episode of TB disease in a TB patient treated successfully for at least 6 months (i.e. smear or sputum culture was negative at the end of the 
treatment period), but developed subsequently active TB. If this new TB episode is due to the same strain as the previous TB episode, this is considered TB relapse, otherwise it is considered TB “recurrence”. Due to the variable definitions for reinfection, recurrence, and relapse in the literature, we opted to define broadly relapse + recurrence as simply “reinfection”.

A recent meta-analysis reported that the risk of TB reinfection is higher (RR of 1.80 95\% CI: 1.40-2.30) among those with DM compared to those without DM [53]. This effect size was accordingly incorporated in the TB-DM model. 


\section{ADDITIONAL FIGURES}

Figure S4. India demographics. (A) Estimated population size between 1980-2050, compared to the projections by the Population Division of the United Nations (UN) Department of Economic and Social Affairs [5]. (B) Estimated population size by age group between 19802050, compared to the UN projections.
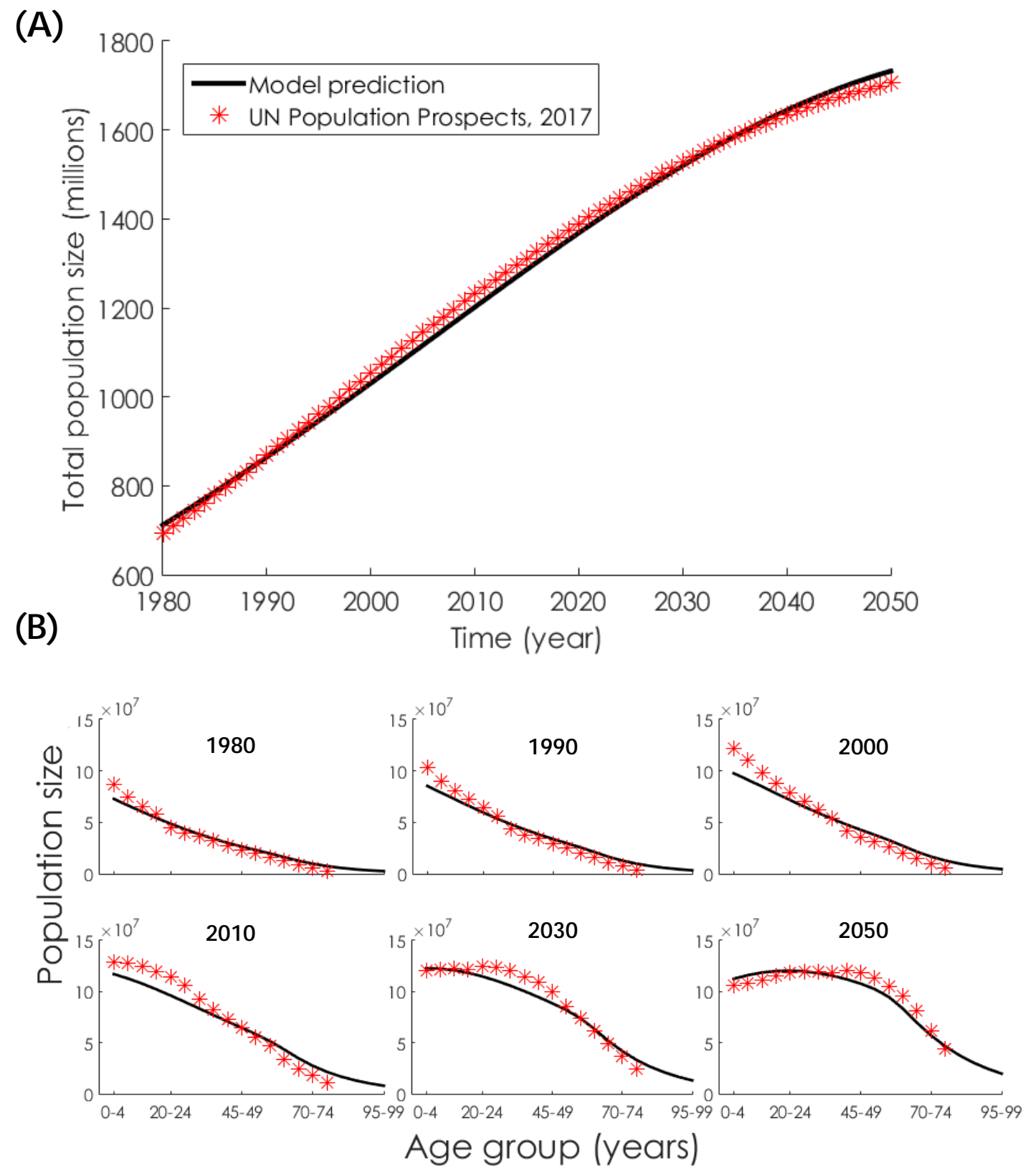
Figure S5. Model projections for the proportion of tuberculosis (TB) disease (A) incident and (B) mortality cases attributed to diabetes mellitus in India at 10 different TB disease incidence rate trajectories. The change in TB incidence rate at 2050, relative to the baseline model scenario, was assumed to range between $-50 \%$ to $+50 \%$.

(A)
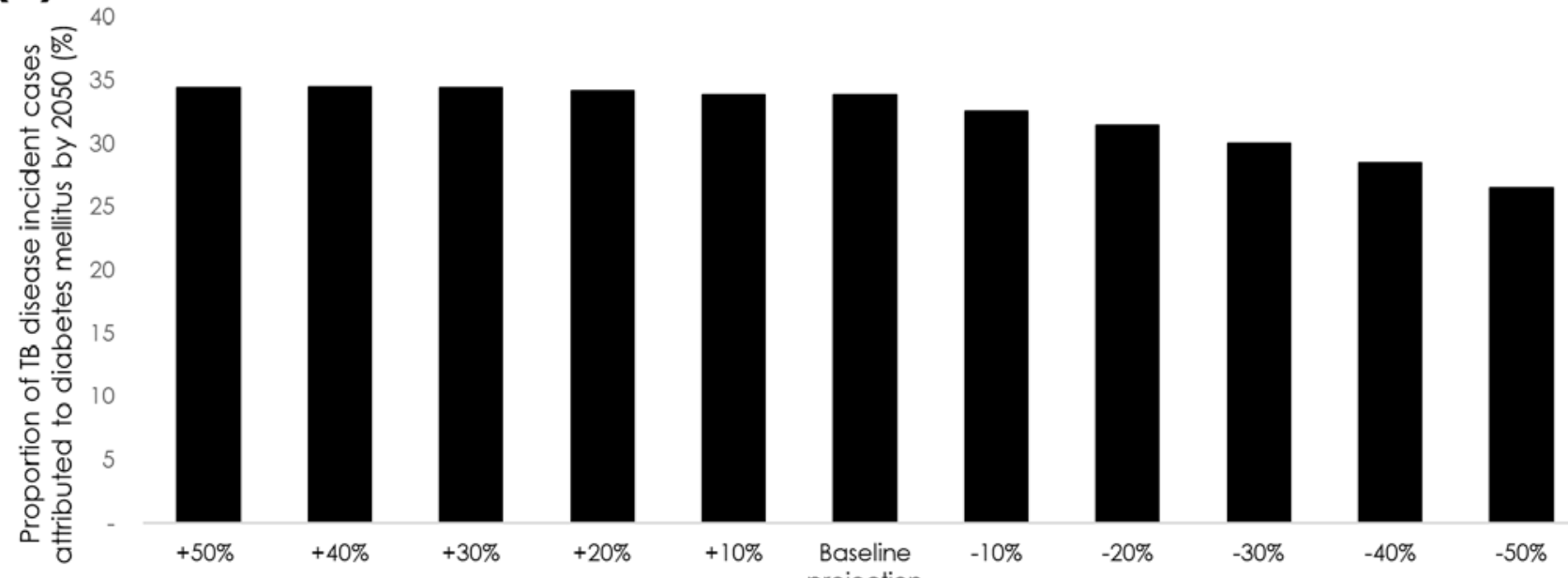

Relative TB incidence rate to baseline model projection at 2050

(B)

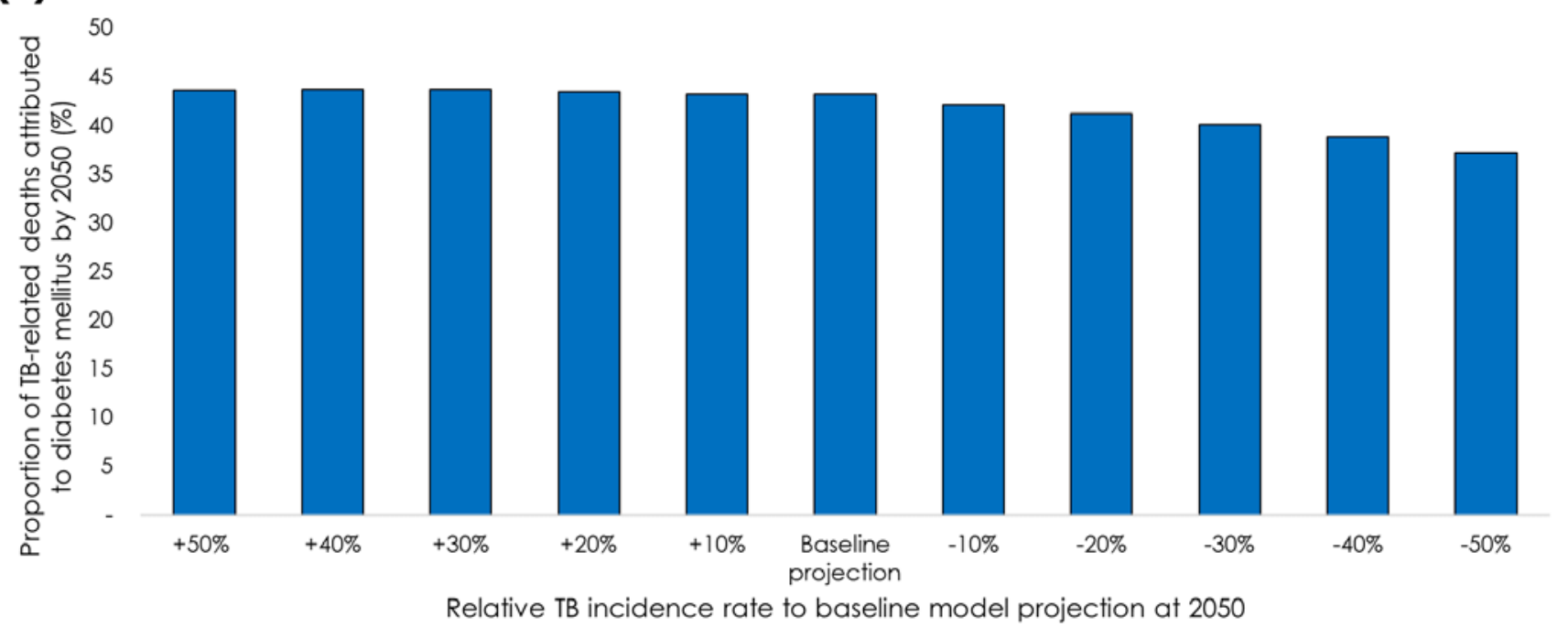


Figure S6. Model predictions for the proportion of tuberculosis (TB) disease incident (solid black line) and mortality (dashes blue line) cases attributed to diabetes mellitus (DM) in India between 1990 and 2050, assuming age-dependency in the proportion of individuals developing latent slow versus latent fast TB infection.

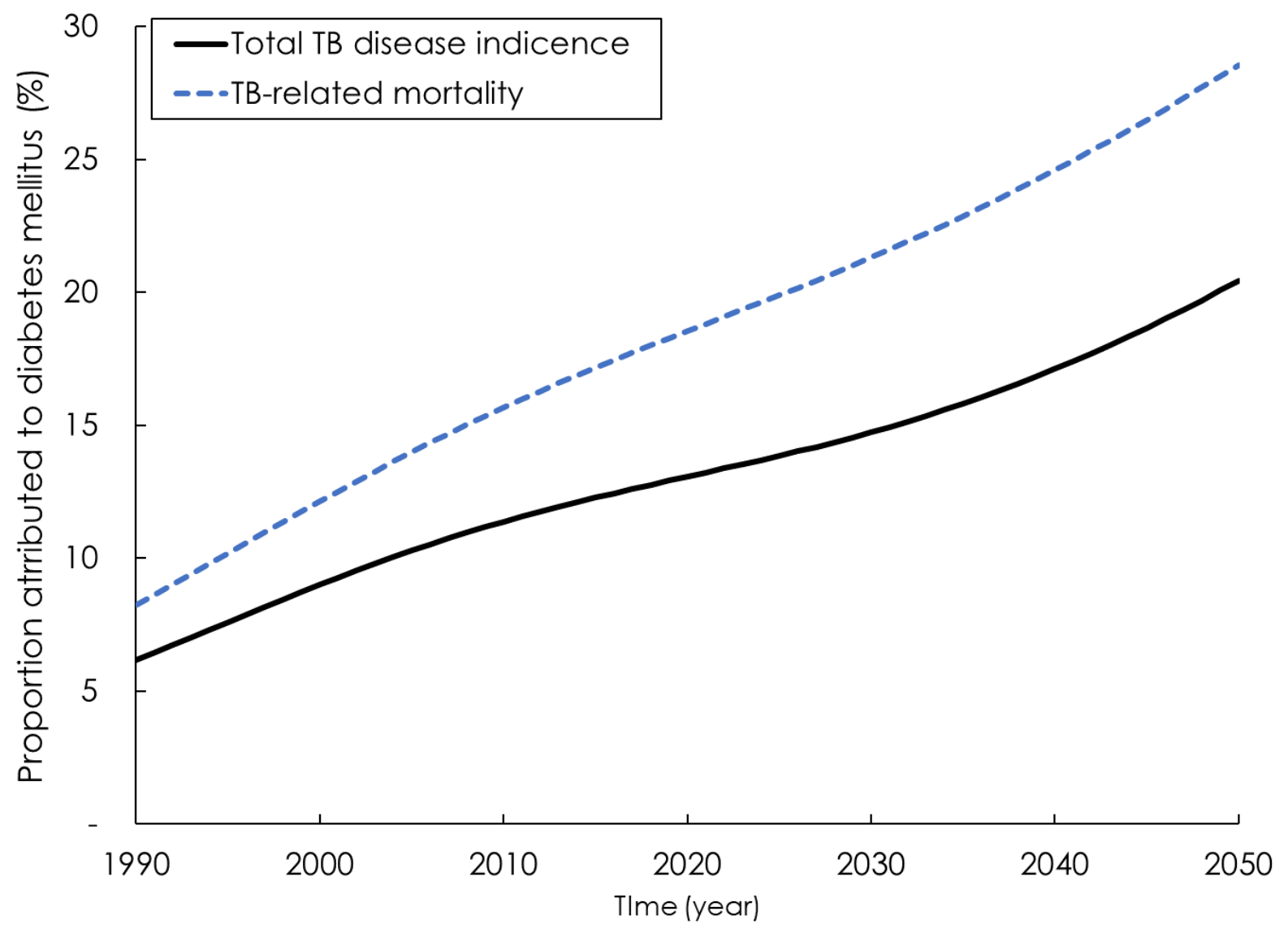


Figure S7. Model predictions for the proportion of tuberculosis (TB) (A) disease incident and (B) mortality cases attributed to diabetes mellitus (DM) in India by 2050, assuming different risk levels of the susceptibility to TB reinfection among individuals $i$ ) latently infected with TB, ii) who successfully completed TB treatment, and iii) both latently infected with TB or who successfully completed TB treatment.

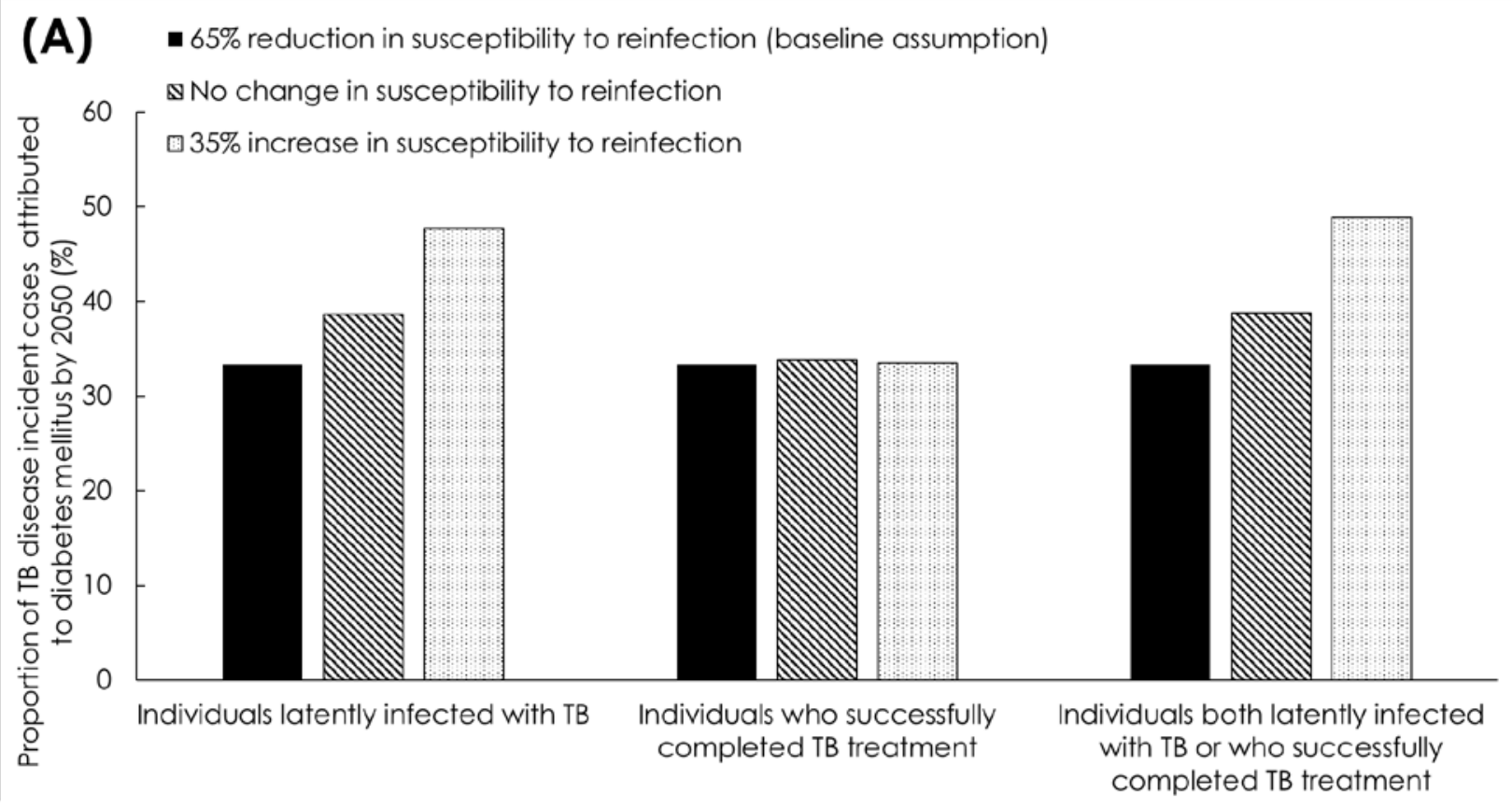

(B) $\quad 65 \%$ reduction in susceptibility to reinfection (baseline assumption) $\mathbb{N}$ No change in susceptibility to reinfection

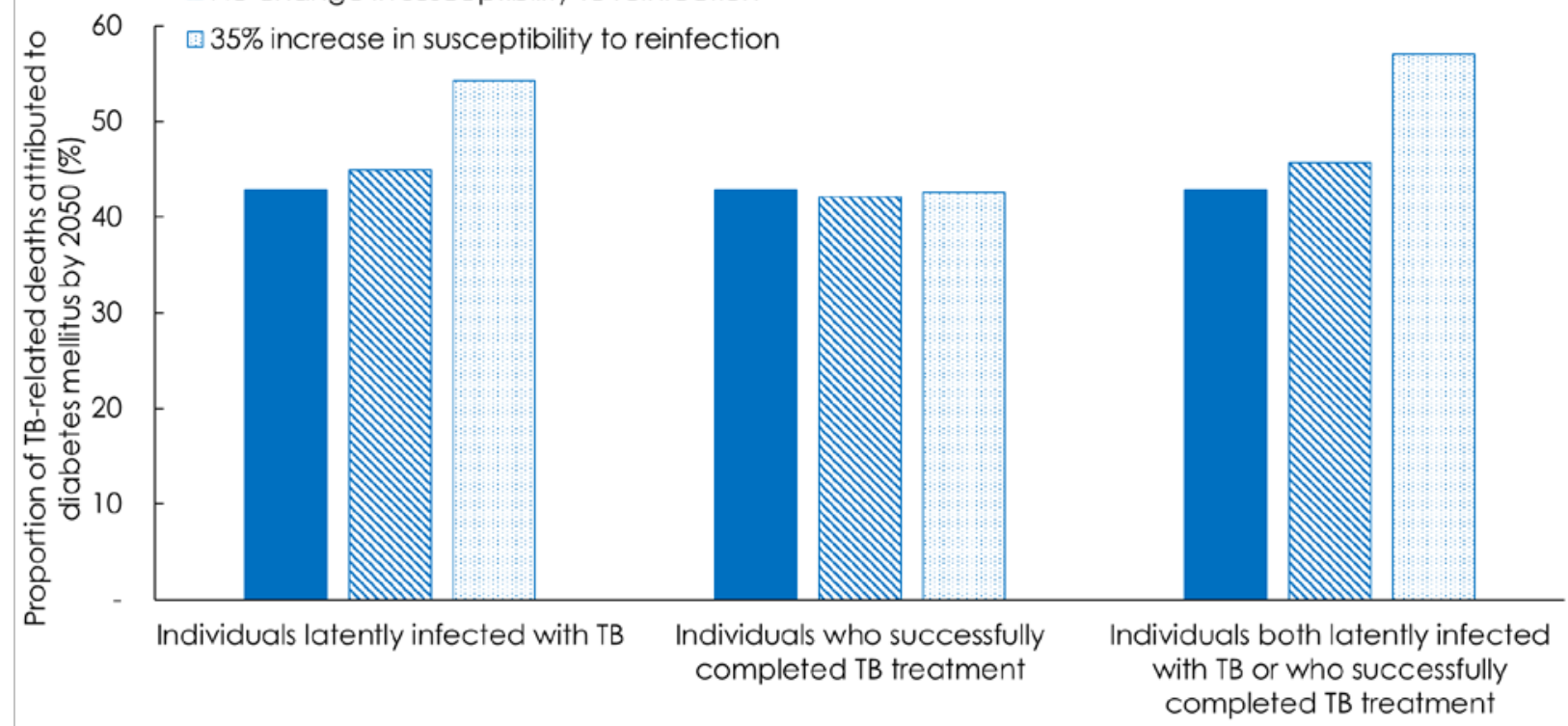


Figure S8. Uncertainty intervals for the proportion of tuberculosis (TB) disease (A) incident and (B) mortality cases attributed to diabetes mellitus in India between 1990 and 2050. The solid red lines represent the mean, while the dashed lines bracket the $95 \%$ uncertainty interval.

(A)

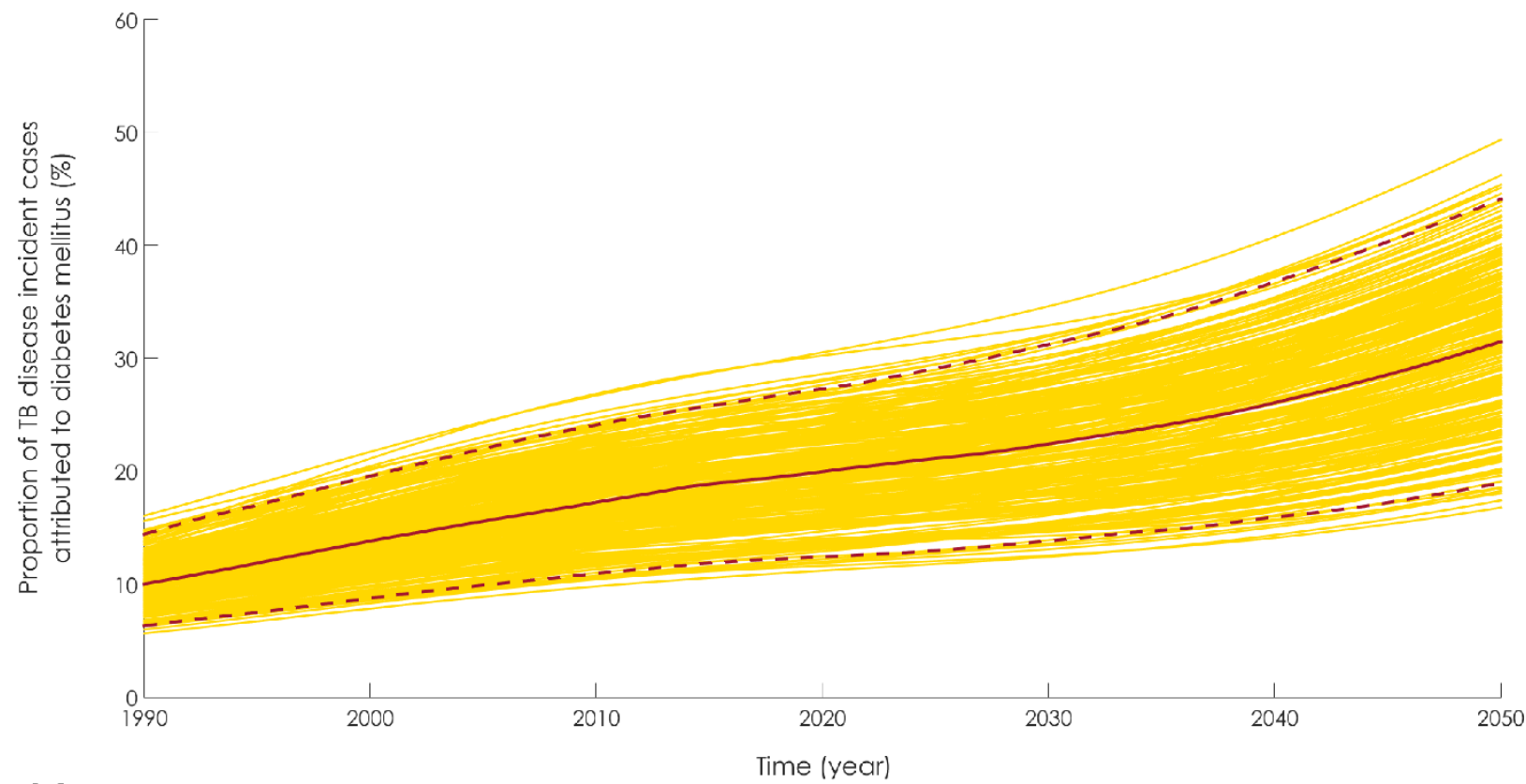

(B)

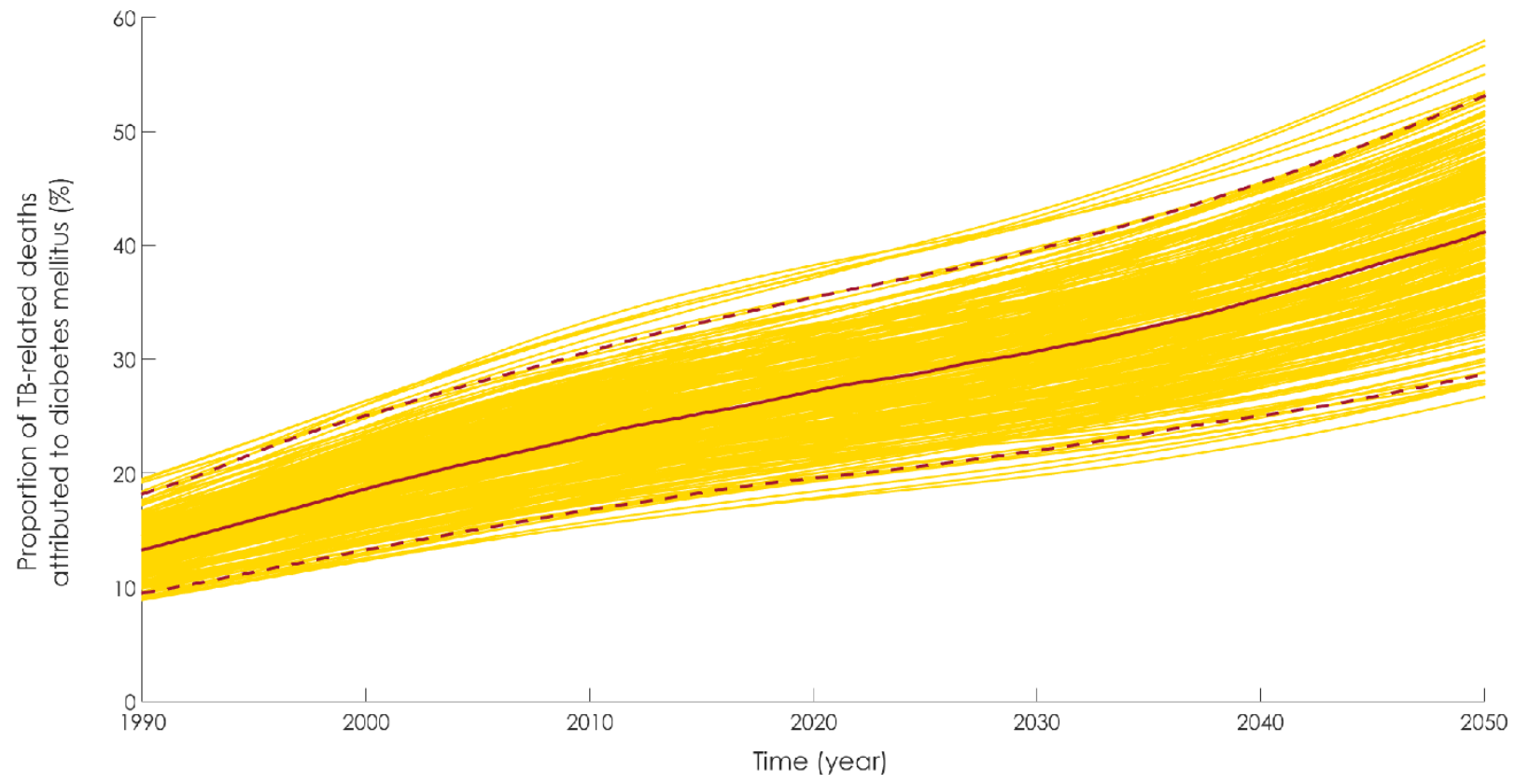


Figure S9. Sensitivity analyses to assess the sensitivity of the proportion of tuberculosis (TB) disease (A) incident and (B) mortality cases attributed to diabetes mellitus in 2050, to variations in the key parameters in the model. Blue bars are based on the lower bound of parameter values (lower bound of the 95\% confidence interval; CI) and red bars are based on the upper bound of parameter values (upper bound of the 95\% CI; Table 1).

(A) Proportion of TB diseae incident cases attributed to diabetes mellitus (\%)

Effect:

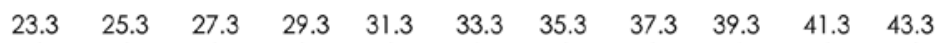
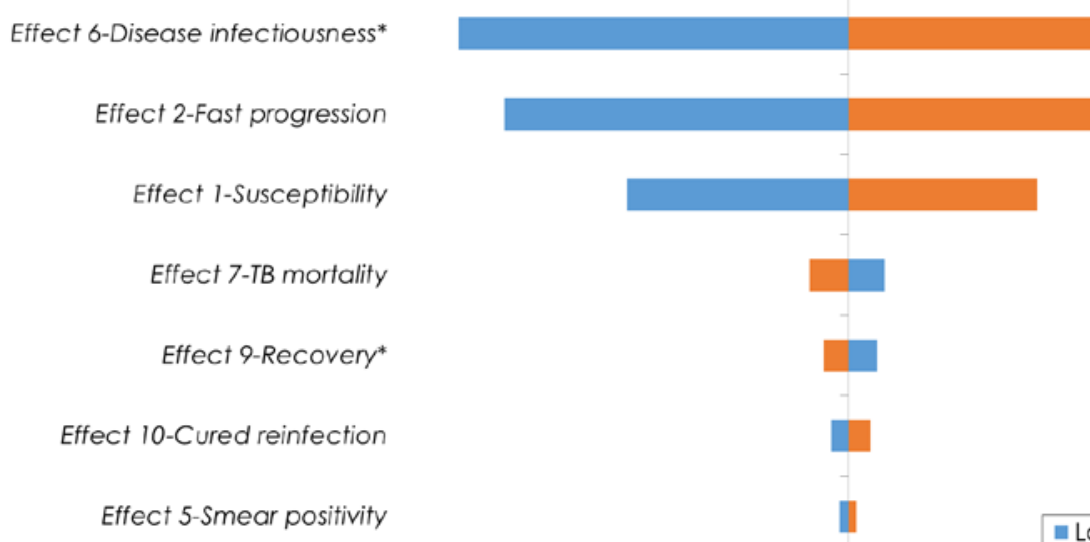

(B)
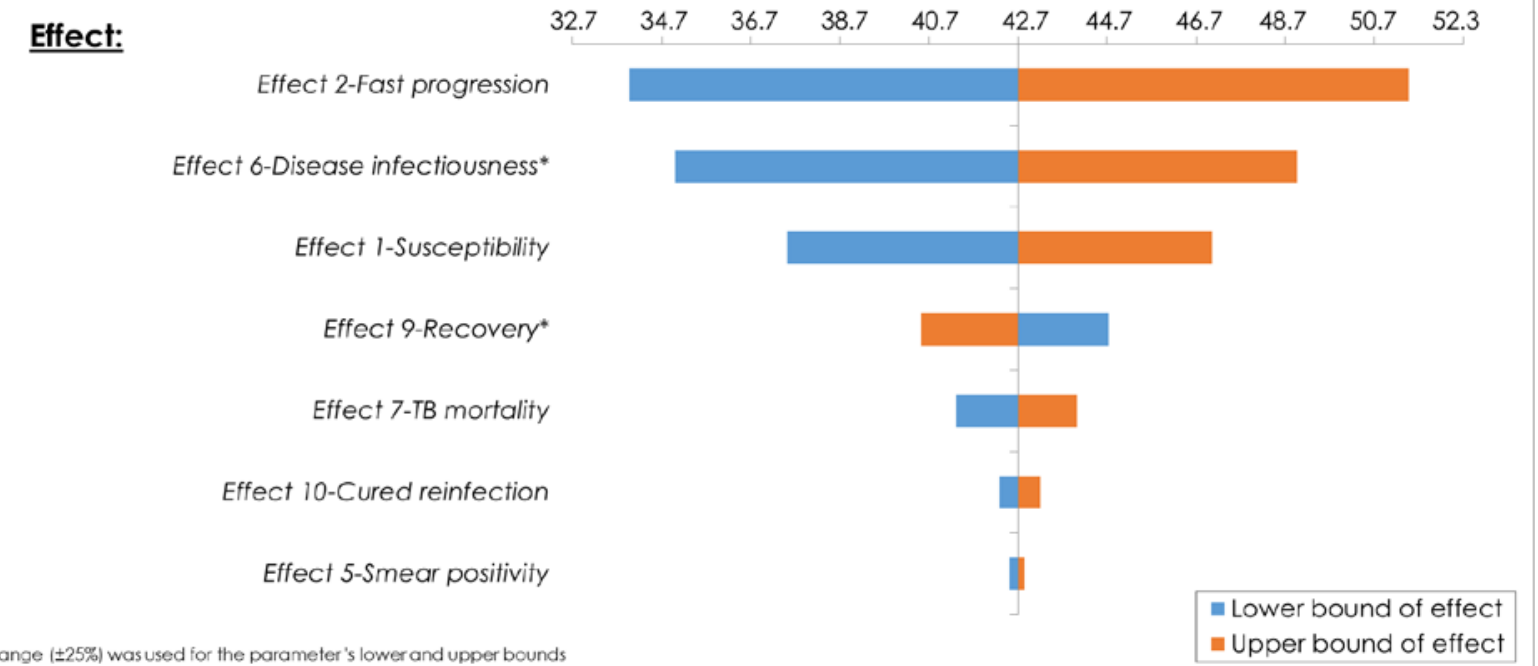


\section{REFERENCES}

1 Awad SF, Dargham SR, Omori R, Pearson F, Critchley JA, Abu-Raddad LJ. Analytical Insights into the Impact of Diabetes Mellitus on Tuberculosis Epidemiology. Under review. 2018.

2 The MathWorks, Inc. MATLAB. The language of technical computing. 8.5.0.197613 (R2015a). Natick, MA, USA: ed: The MathWorks, Inc.; 2015.

3 Abu-Raddad LJ, Sabatelli L, Achterberg JT, Sugimoto JD, Longini IM, Jr., Dye C, et al. Epidemiological benefits of more-effective tuberculosis vaccines, drugs, and diagnostics. Proceedings of the National Academy of Sciences of the United States of America. 2009;106:13980-5.

$4 \quad$ World Health Organization. Global Tuberculosis Control Surveillance, Planning, Financing (available at: http://data.unaids.org/pub/report/2008/who2008globaltbreport en.pdf). Switzerland: World Health Organization, 2008.

5 United Nations Department of Economic and Social Affairs, Population Division, Population Estimates and Projections Section. World population prospects, the 2012 revision. 2012. available: http://esa.un.org/wpp/Excel-Data/population.htm

6 Velicia FJF. On the moments of a Wood Saxon beta distribution. Journal of Physics A: Mathematical and General. 1987.

7 Woods RD, Saxon DS. Diffuse Surface Optical Model for Nucleon-Nuclei Scattering. Physical Review. 1954;95:577-8.

8 World Health Organization. WHO Global Health Observatory Data Repository, (available at: http://apps.who.int/gho/data/node.main). 2017. Accessed.

9 The World Bank. Tuberculosis case detection rate in India (\%, all forms) (Available at: https://data.worldbank.org/indicator/SH.TBS.DTEC.ZS?locations=IN\&year high desc=false; Accessed November, 2017). 2015.

10 International Diabetes Federation. IDF Diabetes Atlas. 7th edition. Brussels, Belgium (Available at:http://www.diabetesatlas.org; accessed on September 2016). 2016.

11 Anjana RM, Deepa M, Pradeepa R, Mahanta J, Narain K, Das HK, et al. Prevalence of diabetes and prediabetes in 15 states of India: results from the ICMR-INDIAB population-based crosssectional study. The lancet Diabetes \& endocrinology. 2017;5:585-96.

12 Dye C, Garnett GP, Sleeman K, Williams BG. Prospects for worldwide tuberculosis control under the WHO DOTS strategy. Directly observed short-course therapy. Lancet. 1998;352:1886-91.

13 Barnett GD, Styblo K. Bacteriological and X-ray status of tuberculosis following primary infection acquired during adolescence or later. Bulletin of the International Union Against Tuberculosis 52:5-16. 1977.

14 Dye C, Williams BG. Eliminating human tuberculosis in the twenty-first century. J R Soc Interface. 2008;5:653-62.

15 Springett VH. Ten-year results during the introduction of chemotherapy for tuberculosis. Tubercle. 1971;52:73-87.

16 World Health Organization. Treatment of tuberculosis: guidelines -4 th ed. (Available at: http://apps.who.int/iris/bitstream/10665/44165/1/9789241547833 eng.pdf, accessed Nov. 2018). 2010.

17 Small PM, Hopewell PC, Singh SP, Paz A, Parsonnet J, Ruston DC, et al. The epidemiology of tuberculosis in San Francisco. A population-based study using conventional and molecular methods. The New England journal of medicine. 1994;330:1703-9.

18 Chang JT, Dou HY, Yen CL, Wu YH, Huang RM, Lin HJ, et al. Effect of type 2 diabetes mellitus on the clinical severity and treatment outcome in patients with pulmonary tuberculosis: a potential 
role in the emergence of multidrug-resistance. Journal of the Formosan Medical Association = Taiwan yi zhi. 2011;110:372-81.

19 Nakagami T, Group DS. Hyperglycaemia and mortality from all causes and from cardiovascular disease in five populations of Asian origin. Diabetologia. 2004;47:385-94.

20 International Diabetes Federation. IDF Diabetes Atlas. 3th edition. Brussels, Belgium (available at: https://www.idf.org/sites/default/files/Diabetes-Atlas-3rd-edition.pdf; accessed on December 2015). 2006.

21 Restrepo BI, Schlesinger LS. Impact of diabetes on the natural history of tuberculosis. Diabetes research and clinical practice. 2014;106:191-9.

22 Lee MR, Huang YP, Kuo YT, Luo CH, Shih YJ, Shu CC, et al. Diabetes Mellitus and Latent Tuberculosis Infection: A Systemic Review and Metaanalysis. Clinical infectious diseases : an official publication of the Infectious Diseases Society of America. 2017;64:719-27.

23 Martinez L, Zhu L, Castellanos ME, Liu Q, Chen C, Hallowell BD, et al. Glycemic Control and the Prevalence of Tuberculosis Infection: A Population-based Observational Study. Clinical infectious diseases : an official publication of the Infectious Diseases Society of America. 2017.

24 Al-Rifai RH, Pearson F, Critchley JA, Abu-Raddad LJ. Association between diabetes mellitus and active tuberculosis: A systematic review and meta-analysis. PLoS One. 2017;12:e0187967. Kim SJ, Hong YP, Lew WJ, Yang SC, Lee EG. Incidence of pulmonary tuberculosis among diabetics. Tubercle and Lung Disease. 1995;76:529-33.

26 Ponce-De-Leon A, Garcia-Garcia Md Mde L, Garcia-Sancho MC, Gomez-Perez FJ, ValdespinoGomez JL, Olaiz-Fernandez G, et al. Tuberculosis and diabetes in southern Mexico. Diabetes care. 2004;27:1584-90.

27 Magee MJ, Foote M, Maggio DM, Howards PP, Narayan KM, Blumberg HM, et al. Diabetes mellitus and risk of all-cause mortality among patients with tuberculosis in the state of Georgia, 2009-2012. Annals of epidemiology. 2014;24:369-75.

28 Duangrithi D, Thanachartwet V, Desakorn V, Jitruckthai P, Phojanamongkolkij K, Rienthong S, et al. Impact of diabetes mellitus on clinical parameters and treatment outcomes of newly diagnosed pulmonary tuberculosis patients in Thailand. International journal of clinical practice. 2013;67:1199-209.

29 Chiang CY, Bai KJ, Lin HH, Chien ST, Lee JJ, Enarson DA, et al. The Influence of Diabetes, Glycemic Control, and Diabetes-Related Comorbidities on Pulmonary Tuberculosis. PLoS ONE. 2015;10:e0121698.

30 Chiang CY, Lee JJ, Chien ST, Enarson DA, Chang YC, Chen YT, et al. Glycemic control and radiographic manifestations of tuberculosis in diabetic patients. PLoS One. 2014;9:e93397.

31 Dooley KE, Tang T, Golub JE, Dorman SE, Cronin W. Impact of Diabetes Mellitus on Treatment Outcomes of Patients with Active Tuberculosis. The American Journal of Tropical Medicine and Hygiene. 2009;80:634-9.

32 Viswanathan V, Kumpatla S, Aravindalochanan V, Rajan R, Chinnasamy C, Srinivasan R, et al. Prevalence of diabetes and pre-diabetes and associated risk factors among tuberculosis patients in India. PLoS One. 2012;7:e41367.

33 Suwanpimolkul G, Grinsdale JA, Jarlsberg LG, Higashi J, Osmond DH, Hopewell PC, et al. Association between diabetes mellitus and tuberculosis in United States-born and foreign-born populations in San Francisco. PLoS One. 2014;9:e114442.

34 Restrepo BI, Fisher-Hoch SP, Smith B, Jeon S, Rahbar MH, McCormick JB, et al. Mycobacterial clearance from sputum is delayed during the first phase of treatment in patients with diabetes. The American journal of tropical medicine and hygiene. 2008;79:541-4. 
Magee M, Kempker R, Kipiani M, Gandhi N, Darchia L, Tukvadze N, et al. Diabetes mellitus is associated with cavities, smear grade, and multidrug-resistant tuberculosis in Georgia. The International Journal of Tuberculosis and Lung Disease. 2015;19:685-92. Hongguang C, Min L, Shiwen J, Fanghui G, Shaoping H, Tiejie G, et al. Impact of diabetes on clinical presentation and treatment outcome of pulmonary tuberculosis in Beijing. Epidemiology \& Infection. 2015;143:150-6.

37 Magee MJ, Bloss E, Shin SS, Contreras C, Huaman HA, Ticona JC, et al. Clinical characteristics, drug resistance, and treatment outcomes among tuberculosis patients with diabetes in Peru. Int J Infect Dis. 2013;17:e404-12.

38 Gil-Santana L, Almeida-Junior JL, Oliveira CA, Hickson LS, Daltro C, Castro S, et al. Diabetes Is Associated with Worse Clinical Presentation in Tuberculosis Patients from Brazil: A Retrospective Cohort Study. PLoS One. 2016;11:e0146876.

39 Wang Q, Ma A, Han X, Zhao S, Cai J, Ma Y, et al. Prevalence of type 2 diabetes among newly detected pulmonary tuberculosis patients in China: a community based cohort study. PLoS One. 2013;8:e82660.

40 Wu Z, Guo J, Huang Y, Cai E, Zhang X, Pan Q, et al. Diabetes mellitus in patients with pulmonary tuberculosis in an aging population in Shanghai, China: Prevalence, clinical characteristics and outcomes. Journal of diabetes and its complications. 2016;30:237-41.

41 Workneh MH, Bjune GA, Yimer SA. Diabetes mellitus is associated with increased mortality during tuberculosis treatment: a prospective cohort study among tuberculosis patients in SouthEastern Amahra Region, Ethiopia. Infect Dis Poverty. 2016;5:22.

42 Reis-Santos B, Locatelli R, Horta BL, Faerstein E, Sanchez MN, Riley LW, et al. Socio-demographic and clinical differences in subjects with tuberculosis with and without diabetes mellitus in Brazil-a multivariate analysis. PLoS One. 2013;8:e62604.

43 Mi F, Tan S, Liang L, Harries AD, Hinderaker SG, Lin Y, et al. Diabetes mellitus and tuberculosis: pattern of tuberculosis, two-month smear conversion and treatment outcomes in Guangzhou, China. Tropical medicine \& international health : TM \& IH. 2013;18:1379-85.

44 Prasad P, Gounder S, Varman S, Viney K. Sputum smear conversion and treatment outcomes for tuberculosis patients with and without diabetes in Fiji. Public Health Action. 2014;4:159-63.

45 Borenstein M, Hedges LV, Higgins J, Rothstein HR. References: Wiley Online Library; 2009.

46 John NN, George JM, Narmadha M. Study on the prevalence and incidence rates of diabetes mellitus in tuberculosis. International Journal of Pharmaceutical, Chemical \& Biological Sciences. 2017;7:401-6.

47 Singla R, Khan N, Al-Sharif N, Ai-Sayegh MO, Shaikh MA, Osman MM. Influence of diabetes on manifestations and treatment outcome of pulmonary TB patients. The international journal of tuberculosis and lung disease : the official journal of the International Union against Tuberculosis and Lung Disease. 2006;10:74-9.

48 Restrepo BI, Fisher-Hoch SP, Crespo JG, Whitney E, Perez A, Smith B, et al. Type 2 diabetes and tuberculosis in a dynamic bi-national border population. Epidemiology and infection. 2007;135:483-91.

49 Wang CS, Yang CJ, Chen HC, Chuang SH, Chong IW, Hwang JJ, et al. Impact of type 2 diabetes on manifestations and treatment outcome of pulmonary tuberculosis. Epidemiology and infection. 2009;137:203-10.

50 Wang JY, Lee LN, Hsueh PR. Factors changing the manifestation of pulmonary tuberculosis. The international journal of tuberculosis and lung disease : the official journal of the International Union against Tuberculosis and Lung Disease. 2005;9:777-83.

51 Baker MA, Harries AD, Jeon CY, Hart JE, Kapur A, Lönnroth K, et al. The impact of diabetes on tuberculosis treatment outcomes: A systematic review. BMC Medicine. 2011;9:81-. 
52 Faurholt-Jepsen D, Range N, PrayGod G, Jeremiah K, Faurholt-Jepsen M, Aabye MG, et al. Diabetes is a strong predictor of mortality during tuberculosis treatment: a prospective cohort study among tuberculosis patients from Mwanza, Tanzania. Tropical Medicine \& International Health. 2013;18:822-9.

53 Huangfu P, Ugarte-Gil C, Golub J, Pearson F, Critchley J. The effects of diabetes on tuberculosis treatment outcomes: an updated systematic review and meta-analysis Under Review. 2017.

54 Mboussa J, Monabeka H, Kombo M, Yokolo D, Yoka-Mbio A, Yala F. [Course of pulmonary tuberculosis in diabetics]. Rev Pneumol Clin. 2003;59:39-44.

55 Tatar D, Senol G, Cosar D, Ozacar R, Halilcolar H. Patterns of drug resistance in pulmonary tuberculosis cases in the Izmir district, Turkey. New Microbiol. 2009;32:31-7. Jimenez-Corona ME, Cruz-Hervert LP, Garcia-Garcia L, Ferreyra-Reyes L, Delgado-Sanchez G, Bobadilla-Del-Valle $M$, et al. Association of diabetes and tuberculosis: impact on treatment and post-treatment outcomes. Thorax. 2013;68:214-20.

57 Sulaiman SA, Khan AH, Muttalif AR, Hassali MA, Ahmad N, Iqubal MS. Impact of diabetes mellitus on treatment outcomes of tuberculosis patients in tertiary care setup. Am J Med Sci. 2013;345:321-5.

58 K VN, Duraisamy K, Balakrishnan S, M S, S JS, Sagili KD, et al. Outcome of tuberculosis treatment in patients with diabetes mellitus treated in the revised national tuberculosis control programme in Malappuram District, Kerala, India. PLoS One. 2013;8:e76275.

59 Viswanathan AA, Gawde NC. Effect of type II diabetes mellitus on treatment outcomes of tuberculosis. Lung India : official organ of Indian Chest Society. 2014;31:244-8.

60 Alo A, Gounder S, Graham SM. Clinical characteristics and treatment outcomes of tuberculosis cases hospitalised in the intensive phase in Fiji. Public Health Action. 2014;4:164-8.

61 Viswanathan V, Vigneswari A, Selvan K, Satyavani K, Rajeswari R, Kapur A. Effect of diabetes on treatment outcome of smear-positive pulmonary tuberculosis--a report from South India. Journal of diabetes and its complications. 2014;28:162-5.

62 Cavanaugh J, Viney K, Kienene T, Harley D, Kelly P, Sleigh A, et al. Effect of diabetes on tuberculosis presentation and outcomes in Kiribati. Tropical Medicine \& International Health. 2015.

63 Ambrosetti M, Besozzi G, Codecasa LR, Farris B, Nutini S, Saini L, et al. The Italian AIPO study on tuberculosis treatment results, report 1997. National AIPO "Tuberculosis" Study Group. Monaldi Arch Chest Dis. 1999;54:407-12.

64 Ambrosetti M, Besozzi G, Codecasa LR, Farris B, Nutini S, Saini L, et al. The Italian AIPO study on tuberculosis treatment results, report 1995. National AIPO "Tuberculosis" Study Group. Monaldi Arch Chest Dis. 1999;54:49-54.

65 Ambrosetti M, Besozzi G, Farris B, Nutini S, Saini L, Casali L, et al. The Italian AIPO study on tuberculosis treatment results, report 1996. National AIPO "Tuberculosis" Study Group. Associazione Italiana Pneumologi Ospedalieri. Monaldi Arch Chest Dis. 1999;54:237-41. Centis R, Ianni A, Migliori GB. Evaluation of tuberculosis treatment results in Italy, report 1998. Tuberculosis section of the National AIPO Study Group on Infectious Disease and the SMIRA Group. Monaldi Arch Chest Dis. 2000;55:293-8.

67 Centis R, Migliori GB, Tuberculosis Study Group. National AIPO, Group S, National Tuberculosis Project ISdS. Evaluation of tuberculosis treatment results in Italy, report 1999. Monaldi Arch Chest Dis. 2002;57:297-305. 